\title{
Memory-Guided Sensory Comparisons in the Prefrontal Cortex: Contribution of Putative Pyramidal Cells and Interneurons
}

\author{
Cory R. Hussar and Tatiana Pasternak \\ Department of Neurobiology and Anatomy, University of Rochester, Rochester, New York 14642
}

Comparing two stimuli that occur at different times demands the coordination of bottom-up and top-down processes. It has been hypothesized that the dorsolateral prefrontal (PFC) cortex, the likely source of top-down cortical influences, plays a key role in such tasks, contributing to both maintenance and sensory comparisons. We examined this hypothesis by recording from the PFC of monkeys comparing directions of two moving stimuli, S1 and S2, separated by a memory delay. We determined the contribution of the two principal cell types to these processes by classifying neurons into broad-spiking (BS) putative pyramidal cells and narrow-spiking (NS) putative local interneurons. During the delay, BS cells were more likely to exhibit anticipatory modulation and represent the remembered direction. While this representation was transient, appearing at different times in different neurons, it weakened when direction was not task relevant, suggesting its utility. During S2, both putative cell types showed comparison-related activity modulations. These modulations were of two types, each carried by different neurons, which either preferred trials with stimuli moving in the same direction or trials with stimuli of different directions. These comparison effects were strongly correlated with choice, suggesting their role in circuitry underlying decision making. These results provide the first demonstration of distinct contributions made by principal cell types to memory-guided perceptual decisions. During sensory stimulation both cell types represent behaviorally relevant stimulus features contributing to comparison and decision-related activity. However in the absence of sensory stimulation, putative pyramidal cells dominated, carrying information about the elapsed time and the preceding direction.

\section{Introduction}

Active observers are often faced with the task of comparing visual motion across time. In the laboratory, such tasks often consist of subjects comparing the direction or speeds of two moving stimuli separated by a brief delay. To perform such tasks, subjects must be able to not only identify the direction and/or speed of these stimuli but also retain them in memory to be retrieved at the time of comparison. Thus, the underlying circuitry requires the involvement of cortical regions subserving motion processing, maintenance, attention, and decision making. Two reciprocally interconnected cortical regions relevant to these tasks are motion-processing area MT and the dorsolateral region of the prefrontal cortex (PFC) associated with sensory maintenance and executive control (Barbas, 1988; Miller and Cohen, 2001; Petrides and Pandya, 2006). Our recent work showed that MT neurons, in

\footnotetext{
Received Oct. 10, 2011; revised Dec. 2, 2011; accepted Jan. 2, 2012.

Author contributions:T.P. designed research; C.R.H. and T.P. performed research;C.R.H. contributed unpublished reagents/analytic tools; C.R.H. analyzed data; C.R.H. and T.P. wrote the paper.

This work was supported by National Eye Institute Grants R01 EY11749 and P30 EY01319 (Center for Visual Science). We thank Marc Mancarella for technical assistance and Martin Gira for help with electronics. We also very grateful to Albert Compte, Greg DeAngelis, Ben Hayden, Klaus Wimmer, and Mark Zarella for comments and useful suggestions on the manuscript.

The authors declare no competing financial interests.

Correspondence should be addressed to Tatiana Pasternak, Department of Neurobiology and Anatomy, Box 603, University of Rochester, Rochester, NY 14642. E-mail: tania@cvs.rochester.edu.

DOI:10.1523/JNEUROSCI.5135-11.2012

Copyright $\odot 2012$ the authors $\quad 0270-6474 / 12 / 322747-15 \$ 15.00 / 0$
}

addition to exhibiting direction-selective (DS) responses to motion, carried some stimulus-related activity during the delay, and their responses during the comparison phase of the task reflected the previously presented direction (Zaksas and Pasternak, 2006; Lui and Pasternak, 2011).

While the presence of direction selectivity in the PFC suggest bottom-up signals provided by MT (Zaksas and Pasternak, 2006), the origin and the nature of memory- and comparisonrelated activity during motion discrimination tasks remains largely unexplored. Because top-down modulations recorded in sensory neurons during such tasks are thought to arise in the PFC (Miller and Cohen, 2001), characterization of memory and decision-related activity in the PFC will elucidate not only its role in comparison tasks but also the nature of top-down influences the PFC may exert on motion-processing neurons. To address this problem, we focused on the identity of neurons likely to provide sensory neurons with such influences during a motion discrimination task. Anatomical evidence points to excitatory pyramidal cells as a source of the top-down influences and to inhibitory interneurons contributing more to local processing (Rockland, 1997; Markram et al., 2004). In our analysis we took advantage of differences in the temporal dynamics of action potentials between these two classes of neurons, with pyramidal neurons having broader action potentials compared to the relatively narrow spikes characteristic of inhibitory interneurons (Connors and Gutnick, 1990; Gray and McCormick, 1996; Nowak et al., 2003). We used these differences to identify the two 
cells groups as broad-spiking (BS) putative pyramidal and narrow-spiking (NS) putative interneurons.

In a recent study we have shown that while both cell types exhibited comparable direction selectivity to behaviorally relevant visual motion during direction discrimination, NS cells were much more sensitive to the behavioral context, drastically reducing their selectivity when direction was not relevant to the task (Hussar and Pasternak, 2009). Here, we compared the behavior of the two groups during maintenance and comparison stages of the same task. We found that during the delay, BS cells were more likely than NS cells to carry anticipatory and stimulus selective activity, and this activity was affected by the behavioral context. During the comparison stimulus, responses of both cell types reflected similarities and differences between the two stimuli being compared. In individual neurons, these comparison effects were highly correlated with activity predictive of the perceptual report, suggesting their utilization in decision making. Our results provide the first demonstration of distinct contributions made by putative pyramidal neurons and interneurons to memory-related and other task-related activity during sensory comparisons.

\section{Materials and Methods}

\section{Subjects}

We recorded from the PFC of two adult male rhesus macaque monkeys. All experiments were carried out in accordance with the guidelines published in the National Institutes of Health Guide for the Care and Use of Laboratory Animals and approved for use by the University of Rochester Committee for Animal Research (Rochester, NY).

\section{Visual stimuli}

Visual stimuli have been described in previous reports (Hussar and Pasternak, 2009, 2010). Briefly, the animals viewed random-dot stimuli, $4^{\circ}$ in diameter, presented at the fovea on a 19 inch monitor (Ilyama Vision Master Pro 513, $75 \mathrm{~Hz}$ refresh rate) and placed at a distance of $57 \mathrm{~cm}$. The stimuli consisted of coherently moving random dots that subtended $0.03^{\circ}$ of visual angle with a luminance of $15 \mathrm{~cd} / \mathrm{m}^{2}$. The dot density was set to $4.7 \mathrm{dots} / \mathrm{deg}^{2}$. During the direction discrimination task, stimulus speed was set to either 2 or $4^{\circ} /$ s. During the speed discrimination task, the base speed was matched to that day's direction discrimination task. During the passive fixation task, the stimuli, their duration, and other parameters were identical to those used during the relevant discrimination task.

\section{Behavioral tasks}

Direction discrimination task. The structure of this task is outlined in Figure $1 A$, top). Each trial began with the presentation of a small fixation target. Animals initiated each trial by holding fixation on this square $\left( \pm 1.5^{\circ}\right)$ for $1000 \mathrm{~ms}$ and were required to maintain fixation throughout the duration of the trial. If at any point in the trial the monkeys stopped fixating, the trial was discontinued and repeated after a $3 \mathrm{~s} \mathrm{time} \mathrm{out} \mathrm{signaled}$ by a distinct tone. The fixation target was extinguished at the end of each trial and animals reported whether the two stimuli moved in the same or different directions by pressing one of two adjacent response buttons: the right button to report the same directions and the left button to report different directions. During each session we presented a range of direction differences between S1 and S2, bracketing animals' measured thresholds (Fig. $1 B$ ). All "different" direction trials (D-trials) included stimuli moving in the preferred and antipreferred $\left(180^{\circ}\right.$ away from preferred) directions (see Direction-selective responses to motion) and a set of offset directions (10$90^{\circ}$ relative to preferred or antipreferred). These offset directions were presented with equal probability during both S1 and S2. "Same" direction trials (S-trials) consisted of both S1 and S2 stimuli moving in the preferred, antipreferred, or any of the offset directions. The speeds of S1 and S2 were set to the base speed used in the speed discrimination task (see next section, Speed discrimination task) and did not vary.

Speed discrimination task. During the speed discrimination task, signaled by a unique fixation target (Fig. $1 \mathrm{~A}$, small triangle), S1 and S2 moved either at the same or different speeds (Fig. 1 A, bottom plots). The monkeys were rewarded for reporting the two speeds as same or different by pressing one of two response buttons. During this task, we measured the accuracy of speed discriminations by varying speed differences between S1 and S2. Thresholds were measured with the method of constant stimuli in which each trial contained a S1 or S2 moving at the base speed ( 2 or $4 \%$ ) and a comparison stimulus moving at the same or different speed. Differences in speed, chosen to bracket the threshold, ranged between 10 and $300 \%$ (e.g., $2 \%$ was compared to speeds ranging from $2.2 \%$ to $16 \%$ ). The directions of $\mathrm{S} 1$ and $\mathrm{S} 2$ were the same within a given trial but varied randomly from trial-to-trial between a neuron's preferred and antipreferred direction.

Passive fixation task. Animals were cued with a small " $\mathrm{x}$ " fixation point, identifying the passive task condition (Fig. 1, middle plots). During this task, timing and stimuli were identical to the direction discrimination tasks. However, animals were rewarded for maintaining fixation throughout the presentation of both S1 and S2 stimuli, and no button response was required.

\section{Physiological recordings}

Recording locations (Fig. $1 D$ ) were identified from structural MRI scans. Data from a subset of these locations has been previously analyzed (Hussar and Pasternak, 2009, 2010). All recordings were performed using a single tungsten glass-coated microelectrode (0.5-3 M $\Omega$; Alpha Omega Engineering). Electrodes were positioned over the chamber-enclosed craniotomy using a cilux grid with $1 \mathrm{~mm}$ spaced openings (Crist Instruments). Custom-made steel guide tubes were inserted into the hole chosen for each day's recording to provide structural stability. Guide tubes were lowered to the dura but did not penetrate. Electrodes were driven through the dura using either a hydraulic micromanipulator (Narashigi Group) or a NAN electrode drive (NAN Instruments).

Cell selection. Cells were selected while the monkey performed a direction discrimination task, during which the directions of S1 and S2 were either $90^{\circ}$ apart or the same. The durations of the two stimuli and the length of the delay were 500 and $1500 \mathrm{~ms}$, respectively. During this task, eight equally spaced motion directions were used. Single units were selected for further recording if they were clearly differentiated from background noise. Once isolated, the neurons were formally evaluated for task-related activity, defined as significant deviation of firing rates at any point in the trial from baseline activity (Wilcoxon sign-rank test, $p<$ 0.01). As baseline we chose activity during a $200 \mathrm{~ms}$ bin of fixation centered at $500 \mathrm{~ms}$ leading to trial initiation. This was necessary because we found that many PFC neurons change their firing rates immediately before salient events in the trial (see Time-dependent activity modulation)

Cell classification. Previous work with intracellular recordings identified a relationship between certain metrics of a neuron's extracellularly recorded action potential and underlying morphology (McCormick et al., 1985; Contreras and Palmer, 2003). These studies revealed that action potentials of pyramidal neurons have longer durations than those of interneurons. Subsequently, a number of extracellular recording studies used waveform durations to classify neurons in the PFC and in other cortical regions into putative pyramidal neurons and putative interneurons (Mitchell et al., 2007; Diester and Nieder, 2008; Johnston et al., 2009). In the preceding study in which we examined the dependence of DS activity in the PFC on behavioral relevance, we used waveform durations to classify cells as putative interneurons and pyramidal cells (Hussar and Pasternak, 2009). In that paper we provided a detailed account of the methods used for classifying the two cell types and showed that the distribution of action potential durations for all recorded neurons was significantly bimodal (Hartigan's dip test, $p=0.02$; Hartigan and Hartigan, 1985) (Hussar and Pasternak, 2009, their Fig. $1 H$ ). Since the current analysis is based on recordings from the same neurons, here we provide only a brief summary of this classification. Waveforms were isolated and saved with Plexon sorting software (Plexon). For each neuron, an average waveform was derived and interpolated with a spline fit to a precision of $2.5 \mu$ s (Mitchell et al., 2007), and waveform duration was determined by measuring the time between the trough and the peak. The category boundary was based on the trough of the bimodal distribution at $200 \mu \mathrm{s}$. 

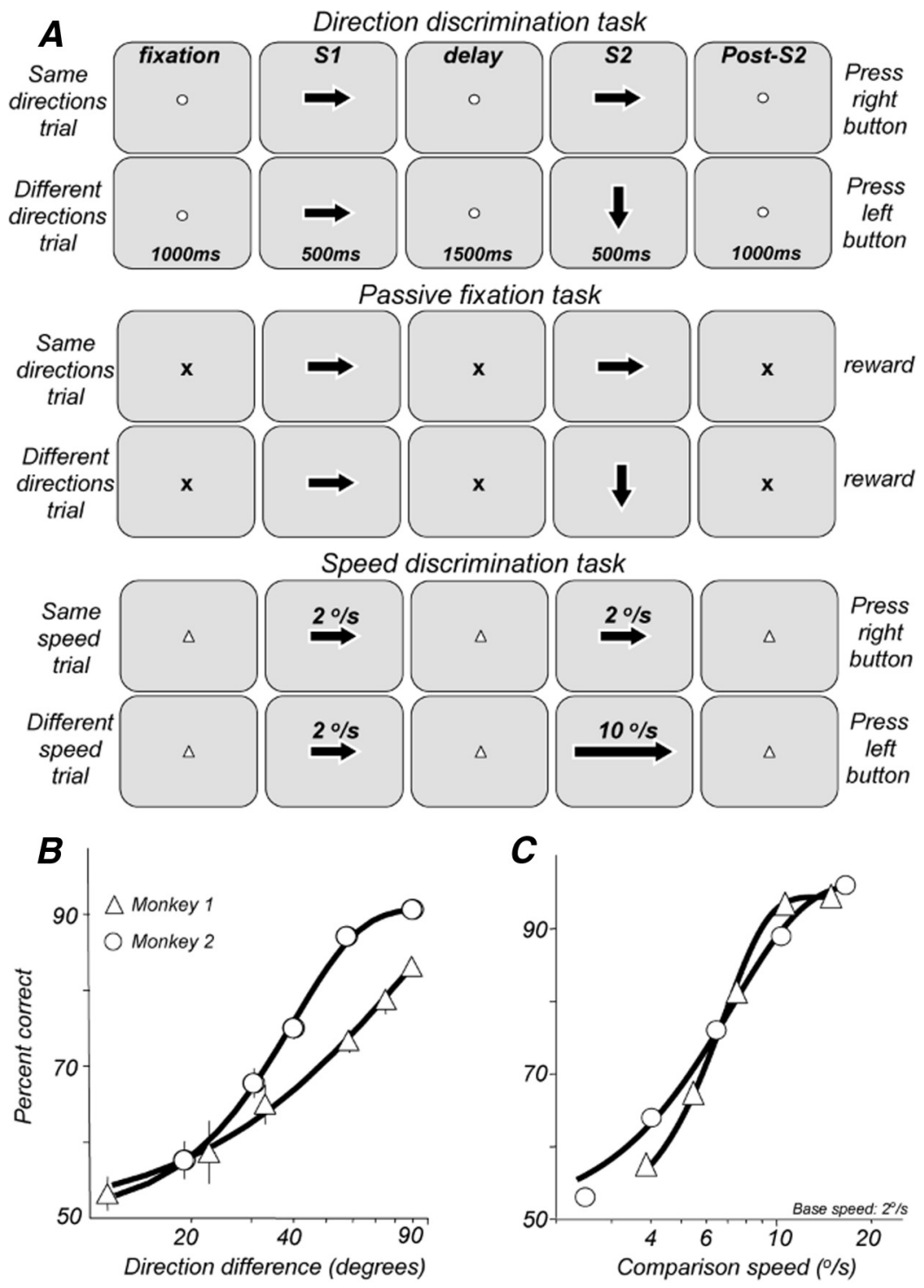

$D$

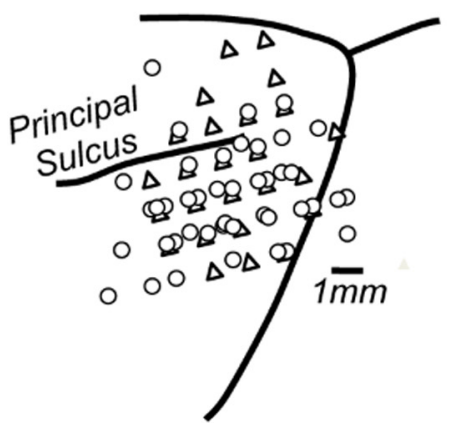

Figure 1. Behavioral tasks, performance, PFC recordingsites. $A$, Diagrams of behavioral tasks. During the direction discriminationtask (top row), animals reported whether the directions of two consecutive random-dot motion stimuli were the same or different by pressing one of two response buttons. The animals were allowed to respond $1000 \mathrm{~ms}$ after the termination of $\$ 2$. During each session, direction difference thresholds were measured byvarying the difference between directionsinS1 and $\$ 2$. Duringthe passivefixationtask (middlerow), stimulus conditions and the timing matched those of the direction task, buttheanimals wereonly required to maintain fixationthroughoutthetrialand automatically receivedareward. During the speed discrimination task (bottom row), the monkeys reported whether two stimuli moved at the same or different speeds. On each trial the two stimuli always moved in the same direction. During each session, speed difference thresholds were measured by varying the differences between $\mathrm{S} 1$ and $\mathrm{S2}$. B, Mean psychometricfunctions for the two monkeys measured during the direction discrimination task. Each function is based on data collected during 159 sessions, each consisting of $150-300$ trials $C$, Representative psychometric functions for the two monkeys measured during a single session of 300 trials during the speed discrimination task. D, Locations of electrode penetrations for all PFC recordings for the two monkeys. Symbols indicating penetrationsfor each animal arethe sameas those used to show their individual behavioral performance (see $\boldsymbol{B}$ and $\boldsymbol{C}$.
This value was used to divide the cells into two classes: neurons with durations $<200 \mu$ s were classified as NS putative inhibitory interneurons, and neurons with waveform durations $>200 \mu$ s were grouped as BS putative pyramidal neurons. Additional care was taken to avoid cell misclassifications (for further details, see Hussar and Pasternak, 2009).

Direction selective responses to motion. Once a given neuron was isolated, its direction selectivity was characterized during a separate task in which one of eight directions of motion would appear during S1 or S2 while the monkeys discriminated $90^{\circ}$ differences in direction. This allowed us to identify the preferred and antipreferred (opposite to preferred) directions for each neuron. Once identified, these two directions were used in all subsequent tasks. During the direction accuracy task, preferred, antipreferred, and offset directions were selected at random to appear during S1 and/or S2. We used Receiver Operator Characteristic (ROC) analysis to quantify direction selectivity.

\section{Analysis of delay activity}

Time-dependent activity modulation. During our task the delay duration was constant and, thus, the appearance of the S2 was highly predictable. We examined whether activity during the delay reflected the predictable structure of our task by evaluating time-dependent changes in firing rates during the second half of the delay period. Using all trials, we computed a delay modulation index (DMI) comparing firing rates (FR) in a $200 \mathrm{~ms}$ window at the middle of the delay $(1250 \mathrm{~ms})$ with activity later in delay $(1900 \mathrm{~ms}):$ DMI $=\left[\mathrm{FR}_{(1900)}-\mathrm{FR}_{(1250)}\right] /$ $\left[\mathrm{FR}_{(1900)}+\mathrm{FR}_{(1250)}\right]$. For this index, positive values are indicative of increasing activity while negative values are indicative of decreasing activity. Significance of individual DMIs was evaluated by a Wilcoxon signed-rank test at $p<0.01$.

Direction selective activity. Periods of DS activity were identified with ROC analysis by comparing activity on trials containing motion in preferred and antipreferred directions presented during S1 (Britten et al., 1992). This analysis provided AROC (area below the ROC curve) values ranging between 0 and 1 . AROC $=0.5$ indicated activity that did not differ between preferred and antipreferred trials, while AROC $>0.5$ indicated higher activity during preferred trials. Conversely, AROC $<0.5$ indicated higher activity on antipreferred trials. Significance of AROCs was evaluated by bootstrap test, shuffling preferred and antipreferred trial labels. This process was repeated 1000 times, generating a distribution of shuffled AROC values. The original AROC value was deemed significant if it fell within the top or bottom $2.5 \%$ of the shuffled distribution $(p<0.05)$. Durations of all directional epochs were evaluated with a sliding significance test. Significance was evaluated in a $100 \mathrm{~ms}$ window stepped in $10 \mathrm{~ms}$ intervals. The onset of a significant directional epoch was taken as the center of the first significant window of at least seven consecutive significant windows. The 
offset of a directional epoch was taken as the center of the first nonsignificant window of at least seven consecutive nonsignificant windows. The duration was calculated as the difference between onset and offset times.

\section{Analysis of responses during $S 2$}

Comparison effects. The direction accuracy task contained two types of trials: in half of the trials S1 and S2 moved in the same directions (Strials), and on the other half they moved in different directions (D-trials). We examined whether responses during the $\mathrm{S} 2$ were affected by the direction of the preceding $\mathrm{S} 1$ by comparing responses during these two types of trials. We reasoned that response modulation by the $\mathrm{S} 1$ direction is a likely reflection of the sensory comparison process required by our task. We termed the differences in responses during the two types of trials a "comparison effect." For this analysis we used trials where the S2 stimulus moved in either the preferred and antipreferred direction. Because the firing rates of DS neurons differed for the two directions, all responses were $Z$-scored before being combined to form two distributions of activity, one for S-trials and one for D-trials. Differences between these distributions were evaluated with ROC analysis. Cells with AROC values of $>0.5$ showed higher activity, and thus preference for S-trials, while cells with AROC $<0.5$ showed higher rates on, and a preference for, D-trials. To identify periods of comparison effects, we used a $100 \mathrm{~ms}$ window slid across spike trains in $10 \mathrm{~ms}$ steps adopting criterion values of $>0.65$ and $<0.35$ and requiring seven consecutive bins at or beyond these values. Neurons with sufficient number of consecutive periods with values of $>0.65$ were categorized into the $S>D$ group (same $>$ different), while neurons meeting the $<0.35$ consecutive criterion formed a $\mathrm{D}>\mathrm{S}$ group (different $>$ same). If a neuron showed activity meeting both criteria, the effect with the longest duration determined categorization.

To compare directly the magnitude and timing of $\mathrm{S}>\mathrm{D}$ and $\mathrm{D}>\mathrm{S}$ effects, AROC values were recalculated taking into account an individual neuron's preferred trial-type (same or different). In this analysis AROC values $>0.5$ correspond to greater activity during the preferred trial type of a neuron (S- or D-trials). Conversely, an AROC $<0.5$ corresponds to weaker activity on a neuron's preferred trial-type. This transformation had the effect of reflecting $\mathrm{D}<\mathrm{S}$ neurons around 0.5 . A separate analysis was performed to determine whether comparison signals scaled with smaller differences in direction between stimuli. AROCs at each level of direction difference were calculated (ranging from $18^{\circ}$ to $90^{\circ}$ ). The effect at the maximal direction difference $\left(90^{\circ}\right)$ was then subtracted from the AROC at each direction difference level.

Choice probability. We also examined whether activity following S2 was predictive of the choices monkeys made on each trial. Because the monkeys sometimes reported S-trials as "different" and sometimes as "same," we were able to compute choice-related activity by comparing firing rates on the trials with identical sensory conditions but different behavioral reports. This analysis, which was also used in a preceding report (Zaksas and Pasternak, 2006), is similar to that introduced by Britten et al. (1996). For this analysis, only cells with at least five S-reports (same directions, right button) and five D-reports (different directions, left button press) were used. Spike counts for all S1/S2 combinations with sufficient right and left responses were $Z$-scored to account for any differences in activity related to the S2 direction. These $Z$-scored spike counts were then combined, creating a distribution of S2 spike counts associated with S- and D-reports. ROC analysis was used to evaluate the differences between the two distributions. This analysis was similar to the analysis of the comparison signals described above. Choice probability (CP) values $>0.5$ are indicative of higher activity before neuron's preferred report (same or different). To identify periods of reliable CP, we slid a $100 \mathrm{~ms}$ window in $10 \mathrm{~ms}$ steps and used criterion values $\geq 0.65$ during seven consecutive bins to categorize neurons as either an S-report neuron or a D-report neuron, depending upon which report showed a greater preceding response.

\section{Results}

The performance of the two monkeys on the direction accuracy task is plotted in Figure $1 B$. The average psychometric functions show that the performance of both monkeys decreased as the difference in direction between S1 and S2 decreased. However, Monkey 1 was less accurate, with direction difference thresholds $38 \%$ higher than those for Monkey 2 (Monkey 1, 50.6 \pm 1.9 ; Monkey 2, $35.5^{\circ} \pm 1.1$ ). This difference in performance between the two animals will be considered below in the context of the comparison effect analysis.

Of the 182 PFC neurons recorded while animals performed the direction discrimination task, 159 neurons showed taskrelated activity ( 91 cells from Monkey 1 and 68 cells from Monkey 2) and were included in the analysis. Recording sites for these neurons are provided in Figure $1 D$, which shows that the majority of sites were concentrated in the prearcuate region (areas $8 \mathrm{ad}$ and $8 \mathrm{av}$ ) and around the posterior portion of the principal sulcus (9/46d and 9/46v), with the larger number of sites in its ventral region (area 9/46v) (Petrides, 2005). The encounter rate of NS $(22 \%, n=35)$ and BS $(78 \%, n=124)$ neurons was uniformly distributed across recording locations.

\section{BS putative pyramidal neurons are more active during the delay}

In the analysis of delay activity we focused on the behavior of NS and BS neurons during the period in the trial where the monkeys both prepare for the upcoming comparison stimulus and maintain information about the direction of the preceding stimulus. The activity of two example neurons (one of each type) on trials where S1 and S2 are moving in the preferred (blue) and antipreferred (red) directions is shown in Figure 2. Following strong DS responses to motion during S1, both neurons showed an increase in activity with time in delay, but this increase was substantially more pronounced for the BS neuron (Fig. 2B). Additionally, both neurons showed periods in the delay with significant differences in activity associated with the preferred and antipreferred directions (solid colored bars along $x$-axis, Wilcoxon sign-rank test $p<0.05$ ). The NS cell (Fig. $2 A$ ) showed a single $\sim 450 \mathrm{~ms}$ period of DS dominated by the antipreferred direction (red bar), i.e., opposite in sign relative to $\mathrm{S} 1$ selectivity. The BS neuron (Fig. 2B) also showed a transient period of DS, but it was dominated by the direction identified as preferred during S1 (blue bar) disappearing toward the end of delay. The activity patterns of these two types of cells are largely representative of the rest of the data presented here. Specifically, both cell types were equally likely to show DS responses to motion during S1 and S2 and showed largely transient DS delay activity. However, as we will show below, BS neurons displayed more pronounced timedependent delay modulation and were more likely to exhibit direction selectivity in their delay activity.

Periods of significant delay activity for all neurons were identified by comparing firing rates recorded during the delay to baseline activity recorded during fixation, $500 \mathrm{~ms}$ before the onset of S1. Given that activity in PFC neurons often changes before salient events (Hussar and Pasternak, 2010), the use of an earlier period during the fixation reduced the possibility of baseline activity being contaminated by these anticipatory rate changes. We performed a running significance test $(p<0.05$, Wilcoxon signed-rank test) in $100 \mathrm{~ms}$ nonoverlapping windows stepped across the delay to identify neurons with significant baselinedeviated activity. The results of this analysis, shown in Figure $3 A$, illustrate a pronounced difference between the two cell groups, particularly later in the delay. Immediately after S1 offset, the two groups of neurons were equally likely to continue to fire above baseline levels ( $\chi^{2}$ test, $p>0.05$ ), likely due to S1 responses extending into the delay. During the first $750 \mathrm{~ms}$ of the delay, the proportion of NS neurons decreased, reaching a plateau with 

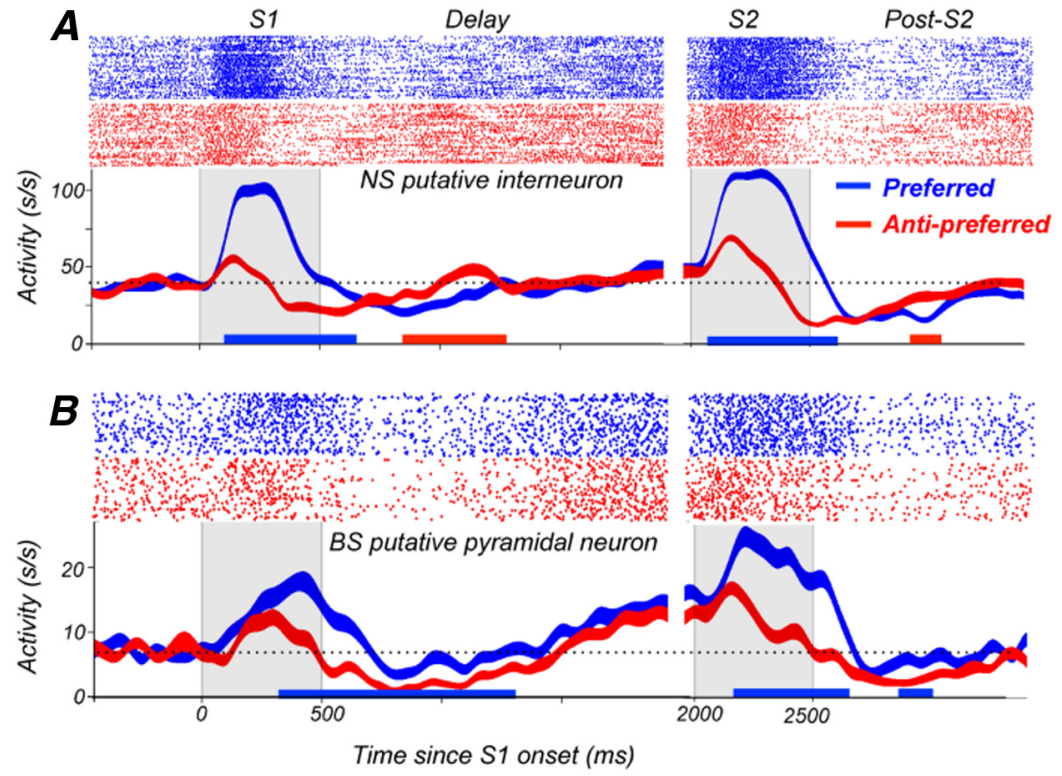

Figure 2. Activity of two example neurons during the direction comparison task Raster plots and average activity for an example NS putative interneuron $(\boldsymbol{A})$ and BS putative pyramidal neuron $(\boldsymbol{B})$. Activity from trials with the $\mathrm{S} 1$ and $\mathrm{S} 2$ stimuli moving in a neuron's preferred and antipreferred directions are indicated by blue and red colors, respectively. Because directions often differed between $\mathrm{S} 1$ and $\mathrm{S} 2$, trials before $\mathrm{S} 2$ onset were selected based on the $\mathrm{S} 1$ direction, while trials after $\mathrm{S} 2$ onset were selected based on the $\$ 2$ direction. Periods of significantly higher activity following $\$ 1$ moving in preferred and antipreferred directions are indicated by blue and red lines, respectively, plotted along the $x$-axis (Wilcoxon sign-rank, $p<0.05$ ).

$\sim 20 \%$ of cells showing significant baseline-deviated activity. Conversely, toward the end of the delay an increasing number of BS neurons began diverging from baseline levels. As a result, during the last $200 \mathrm{~ms}$ of the delay there was a significant difference in the incidence of active neurons between the two groups ( $\chi^{2}$ test, $p=0.03$ ). This disproportionally high number of BS cells active before the onset of $S 2$ suggests these neurons may play a role in preparation for the upcoming sensory stimulus. This possibility is supported by a similarly greater incidence of BS neurons active during the last $100 \mathrm{~ms}$ of fixation, leading to the onset of S1 (NS, 3\%; BS, 25\%; $\chi^{2}$ test, $p=0.001$ ). Such activity differences between the two cell groups were not observed during the S1 (NS $=58 \%$; BS $=60 \% ; \chi^{2}$ test, $\left.p=0.67\right)$ or during S2 (NS $=66 \%$; BS $=71 \% ; \chi^{2}$ test, $\left.p=0.54\right)$. Thus, BS neurons may indeed serve a general function in the preparation for sensory stimuli in PFC. This idea is supported by the recent observation of reduced trial-to-trial variability (Fano factor) in these neurons before stimulus onset, another likely indicator of neuronal task engagement (Hussar and Pasternak, 2010).

While this analysis revealed a higher incidence of active BS neurons during the delay, it provided no information about the time course of this activity. In a recent paper we analyzed delay activity of BS neurons and found that some cells increased activity and some cells decreased their activity with time in delay (Hussar and Pasternak, 2010). Because these patterns of ramping activity largely disappeared during the passive fixation task, the nature of this activity was anticipatory, reflecting task engagement. Here, we compared the patterns of delay activity observed in BS cells to those recorded in NS neurons. To quantify the magnitude and the sign of delay modulation, we calculated a delay modulation index or DMI, comparing activity between the middle and end of the delay (see Materials and Methods). This analysis identified cells with three types of activity for each cell type: neurons with increasing activity (DMI $>0$ ), decreasing activity (DMI $<0$ ), and cells with no significant modulation $(\mathrm{DMI} \sim 0)$. Figure $3 B$ shows the distributions of this index for all BS (top) and NS neurons (bottom). The time courses of activity (baseline subtracted) averaged for each of these three types of delay modulation are shown in Figure $3 C$. The two groups of BS neurons with significant time-dependent modulations, neurons with upward $(n=50)$ and downward $(n=23)$ changes in rates (broken and dotted lines), illustrate the nature of what appears to be preparatory modulation leading to the onset of the S2. In contrast, delay activity in the majority of NS cells showed no significant modulation (77\%), and only a small proportion of cells $(23 \%)$ showed $\operatorname{upward}(n=5)$ or downward $(n=3)$ changes in rates with time in delay (Fig. 3B). This difference in the incidence of delay modulation between NS and BS neurons was significant $\left(\chi^{2}\right.$ test; $p=$ 0.006 ) and suggests an active role for BS neurons in the preparation of the coming comparison process, shared by only a few NS neurons. Supporting this hypothesis is our observation that a significantly smaller percentage of BS neurons exhibited time-dependent delay activity during the passive fixation task (direction task, $n=36 / 57,63 \%$; passive fixation, $11 / 57,19 \% ; \chi^{2}$ test, $p=2.0 \times$ $\left.10^{-6}\right)$. The already small number of NS neurons with timedependent modulation during the direction task became even smaller during passive fixation (direction task, $n=4 / 16=25 \%$; passive fixation, $1 / 16=6 \%$ ). Considering the small number of neurons, it is not surprising that this difference did not reach significance ( $\chi^{2}$ test, $p=0.14$ ).

These results illustrate a striking difference in delay activity between the two groups of cells. The more numerous BS neurons were not only more active than NS cells during the delay, they were also more likely to change their activity in preparation for the upcoming comparison phase of the task.

\section{BS neurons are more likely to represent $\mathrm{S} 1$ direction during the delay}

We previously reported the presence of brief periods of stimulusselective activity in the PFC during the delay in a similar task (Zaksas and Pasternak, 2006) but did not examine the relationship of these signals to directional preferences during S1 or how this selectivity differed between cell types. In the current study, we were particularly interested in potential differences in the nature of stimulus-related delay activity represented by NS and BS neurons and whether this activity reflected direction-selective signals displayed by these cells in response to S1. To identify direction-selective activity, we used ROC analysis to compare activity associated with the preferred and antipreferred directions presented during S1 (see Materials and Methods). The results are presented separately for individual BS (top) and NS (bottom) neurons in Figure 4A. The plot shows DS activity for each neuron during the $\mathrm{S} 1$ and the delay as color-coded AROC values, with smaller and larger values represented by warmer (red) and cooler (blue) colors, respectively. In this analysis, the "preferred direction" was chosen on the basis of each neuron's greater activity during the earliest significant DS epoch. Once identified for a given neuron, this label was maintained for the remainder of the 
A

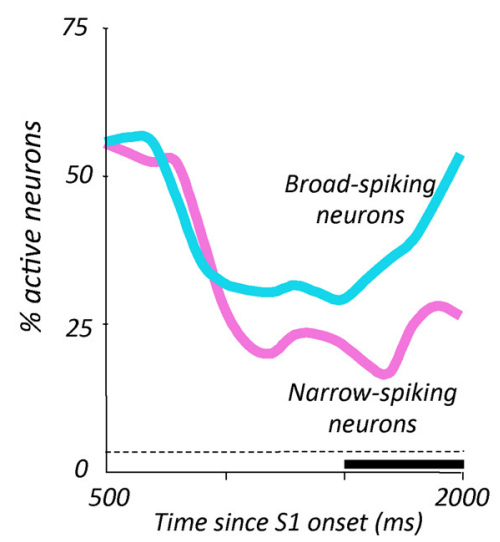

B

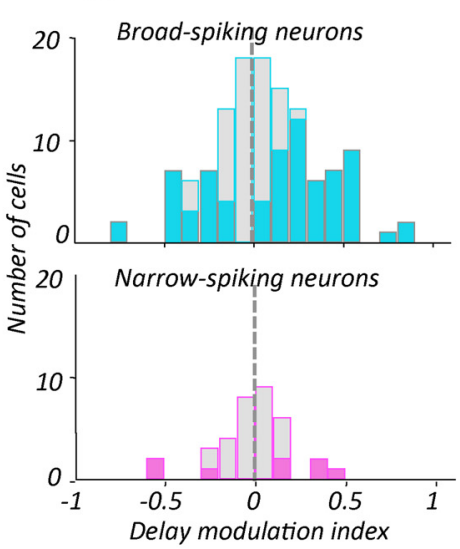

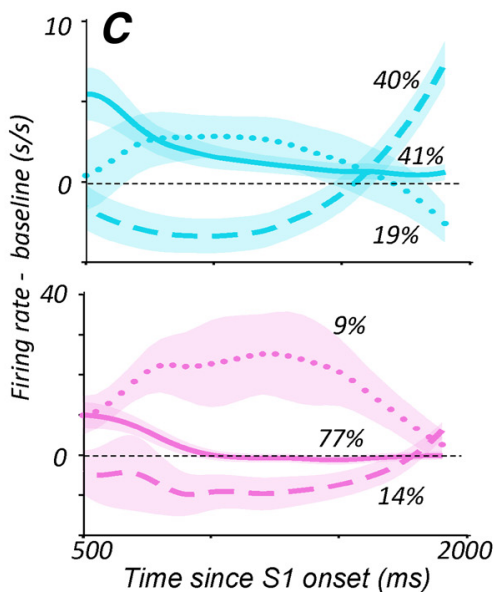

Figure 3. Broad-spiking neurons show anticipatory delay modulation $A$, Incidence of NS $(n=35)$ and $\mathrm{BS}(n=124)$ neurons active (relative to baseline) during the delay. The thick black line signifies the period of significant difference between NS and BS $\left(\chi^{2}\right.$ test, $\left.p<0.05\right)$. The dashed line at $5 \%$ represents significance level expected by chance. $B$, Time-dependent modulation of delay activity for BS (top) and NS (bottom) neurons. Delay modulation index (DMI) $=$ [activity ${ }_{(\text {late delay) }}-$ activity $\left._{\text {(middle delay) }}\right] /\left[\right.$ activity ${ }_{(\text {late delay) }}+$ activity $_{\text {(middle delay) }}$; "late delay", last 200 ms of delay; "middle delay", $200 \mathrm{~ms}$ period centered at $1250 \mathrm{~ms}$. DMI $>0$ indicate higher activity in late delay relative to middle delay; DMI $<0$ indicate lower activity in late delay. Filled colored columns, cells with significant indices (Wilcoxon sign-rank test, $p<0.01$ ); gray columns, cells with nonsignificant values. $C$, Average activity of BS (top) and NS (bottom) of neurons classified on the basis of their DMI as cells with rising activity (broken lines), cells with decreasing activity (dotted lines), and cells with no activity modulation (solid lines). The numbers next to each curve show the incidence of each cell group.
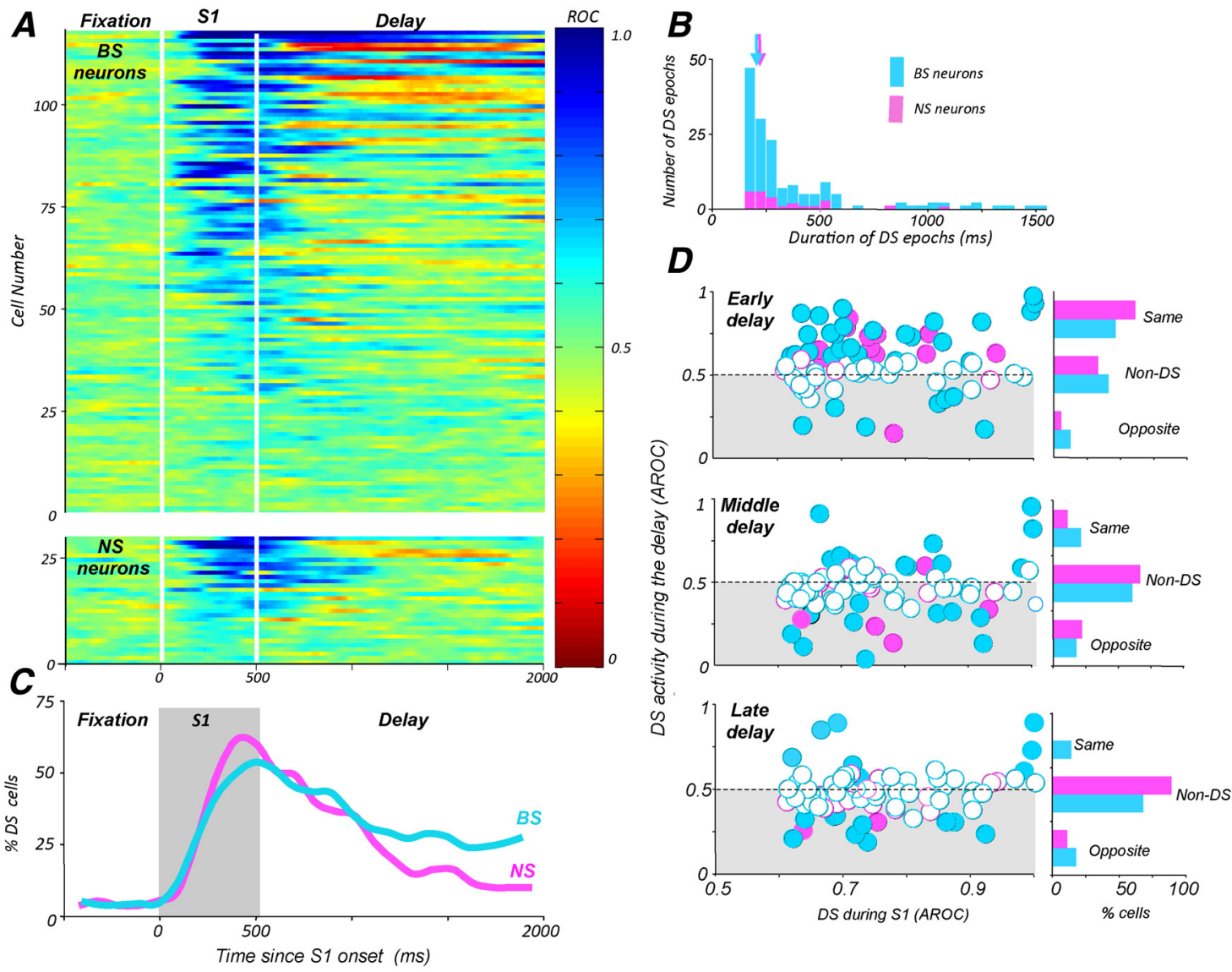

Figure 4. Direction-selective delay activity. $A, D S$ activity during the trial (fixation, $S 1$ and delay) for individual $B S(N=124$, top) and NS ( $N=35$, bottom) neurons quantified by ROC analysis. Scale bar shows ROC values: blue, preferred; red, antipreferred direction. The data were sorted by the average selectivity. $\boldsymbol{B}$, Durations of DS epochs during the delay. Small arrows point to the average duration of DS delay epochs for each cell type (NS, $245 \mathrm{~ms} ; \mathrm{BS}, 242 \mathrm{~ms}$ ). C, Incidence of BS and NS neurons with significant DS (Wilcoxon sign-rank test, $p<0.05$ ). D, Relationship between DS preferences recorded during $S 1$ and during the delay. Only cells with significant DS activity during $S 1$ were used ( $N S, n=18 ; B S, n=56$ ). AROC values computed for each neuron during $S 1$ are plotted against the values computed in early $(500-1000 \mathrm{~ms})$, middle $(1000-1500 \mathrm{~ms})$, and late $(1500-2000 \mathrm{~ms})$ delay. DS during the delay is shown by open $(p>0.05)$ and filled $(p<0.05)$ light blue (BS) or pink (NS) circles. AROC values of $>0.5$ represent DS of the same sign as that of S1, while AROC values of $<0.5$ represents DS opposite in sign to $S 1$. Bar plots to the right are summaries showing the incidence of BS and NS neurons with DS of the same sign (top bars) or opposite sign (bottom bars) as during S1. Middle bars show the incidence of neurons with no DS activity during each delay epoch. 
trial. For example, a consistent direction preference during S1 and throughout the delay would be indicated by a continuous blue line. Conversely, change in preference to the opposite direction between $\mathrm{S} 1$ and delay would be shown by switch from blue to red. The cells were sorted by the average absolute deviation from 0.5 during the task, with neurons showing greater selectivity during the delay near the top of each plot.

Figure $4 A$ illustrates two key features of DS in the delay: its transient nature and inconsistent direction preferences. Significant periods of DS activity were short (Fig. $4 B$ ), rarely extending beyond $500 \mathrm{~ms}$, and were similar between cell types (NS $=245$ $\mathrm{ms} \pm 31.1 ; \mathrm{BS}=242 \mathrm{~ms} \pm 19.6 ; p=0.73$, Wilcoxon signed-rank test). Although the durations of DS periods in NS and BS neurons were similar, they were substantially less common in NS cells, particularly in late delay. During S1 and in early delay, the incidence of DS cells in both groups was similar (Fig. $4 C, \chi^{2}, p>$ 0.05). However, further into the delay, such signals became less common in NS neurons and largely disappeared near the time of S2 onset. This result is consistent with limited activity displayed by these neurons in late delay (see Fig. 3). In contrast, the incidence of DS periods in BS neurons remained relatively constant at $\sim 20-30 \%$, a significantly greater percentage than NS cells (last 200 ms of delay: BS, $n=34,29 \%$; NS, $n=3,9 \%$; $\chi^{2}$ test, $\left.p=0.011\right)$.

The second feature of delay activity is highlighted by the mixture of deep red and blue colors at the top of Figure $4 A$. This pattern is indicative of an absence of consistent preferences for the direction presented during $\mathrm{S}$, since individual neurons sometimes showed stronger activity following the direction preferred during S1, and at other times fired more following the opposite direction. We quantified this observation by directly examining the relationship between DS recorded during the S1 and during the delay (Fig. $4 D$ ). This analysis included only neurons with significant DS during S1 $(p<0.05)$ and was performed separately for the three consecutive $500 \mathrm{~ms}$ delay periods: early (500-1000 ms), middle (1000-1500 ms), and late (1500-2000 $\mathrm{ms})$. In all three graphs, open symbols indicate cells with no reliable DS activity (AROC $\sim 0.5$ ), while filled symbols indicate cells with significant DS activity $(p<0.05)$. The filled data points in the upper half of each graph (AROC $>0.5$ ) indicate significant DS of the same sign as those recorded during S1. Filled data points in the lower half of the plot (shaded) indicate significant DS activity opposite to that during S1 (AROC < 0.5) (see example in Fig. 2 A). The bar plots on the right are summaries of these data, showing the proportions of cells preferring the same (top bars) or opposite (bottom bars) directions as during the S1 and cells with no DS delay activity (middle bars). During the first 500 ms of the delay (Early delay), nearly $50 \%$ of all neurons showed DS activity that matched S1 direction, most likely reflecting S1 responses extending into the delay. The remaining neurons either showed no DS activity or a preference for the opposite direction. In the middle of the delay (Middle delay), the proportion of cells with DS matching S1 direction drastically decreased. while the proportion of cells with no DS activity increased. Less than $20 \%$ of cells of both types showed DS activity, some matching the S1 and some dominated by the antipreferred direction. At the end of the delay (Late delay), the majority of NS and BS cells carried no significant DS signals. Neurons that did display significant delay selectivity were equally likely to show a preference for the same $($ AROC $>0.5)$ or opposite $($ AROC $<0.5)$ direction as the S1. We should note that this analysis underestimates the proportion of cells carrying DS signals, since it excluded neurons that did not respond during S1 or responded but showed no significant DS activity. Thus, the overall proportion of cells with DS periods in the delay was higher $(\sim 25 \%$, see Fig. $4 C)$ than that shown in Figure $4 D$.

In summary, this analysis revealed that in individual neurons the representation of direction during the delay was largely independent of DS activity recorded during S1. While we found no differences in the duration of DS delay activity between the two cell types, this activity was much more likely to be carried by BS putative pyramidal neurons.

\section{Transient direction selective delay signals depend on task demands}

To determine whether the DS delay activity is utilized during the direction task, we examined whether this activity was still present during tasks not requiring direction discriminations. We used two additional tasks, neither of which required direction judgments: the speed discrimination task and the passive fixation task. During the speed task, the monkeys compared speeds of two stimuli moving either at the same or different speeds but always in the same direction. The task structure was identical to the direction task, with the exception of a unique fixation point (Fig. $1 \mathrm{~A}$, bottom diagram). As during the direction task, task difficulty was manipulated by decreasing the differences between the two stimuli and measuring accuracy thresholds. Representative psychometric functions for the two animals show that both monkeys were engaged during the speed discrimination task and this engagement was comparable to that during the direction task (see Fig. 1C). We had sufficient data to compare DS activity of 112 neurons (NS, $n=25$; BS, $n=87$ ) recorded during the direction and the speed tasks.

Figure 5, $A$ and $B$, show the comparison of DS delay activity during in the two tasks. The plots include both cell types, although the small proportion of NS cells (pink symbols) with significant DS during the delay (see Fig. 4C) precluded separate statistical evaluation of their effects. In a previous study (Hussar and Pasternak, 2009), which focused on the effects of task demands on DS responses, we found that DS responses decreased during the speed task and that this reduction was greater in NS neurons. This is illustrated in Figure 5, $A$ and $B$ (S1 plot), showing pronounced decrease in the overall DS during the speed task (all cells, $p=0.002$; BS, $p=0.087$; NS, $p=0.0018$, Wilcoxon signrank test) and that this decrease was significantly greater for NS ( $p=0.01$, Mann-Whitney $U$ test). The scatter plots to the right extend this analysis to delay, identifying cells with DS activity during either of the two tasks. Average AROC calculated for all neurons of both types with significant selectivity in either task, shown in Figure 5A, illustrate that DS during S1 and the last two-thirds of the delay was significantly lower when motion direction was irrelevant to the task. The data in the scatter plots (Fig. 5B) compare these effects on a cell-by-cell basis during the three portions of the delay. In early delay (700-1000 ms), the overall effect was not significant $(p=0.09$; Wilcoxon sign-rank test), although when only cells with DS activity during the direction task (shown by circles) were examined, the drop in DS activity was highly significant $(p=0.0004$; Wilcoxon sign-rank test). With time in delay, the majority of cells showed less DS activity during the speed task (middle delay, 1000-1500 ms, $p=$ 0.02 ; late delay, $1500-2000 \mathrm{~ms}, p=0.02$, Wilcoxon sign-rank test), displaying sensitivity of DS activity represented largely by BS cells to task demands.

The drop in DS delay activity was even more pronounced during passive fixation, when the animals were shown S1 and S2 separated by a delay but were not required to make a response 

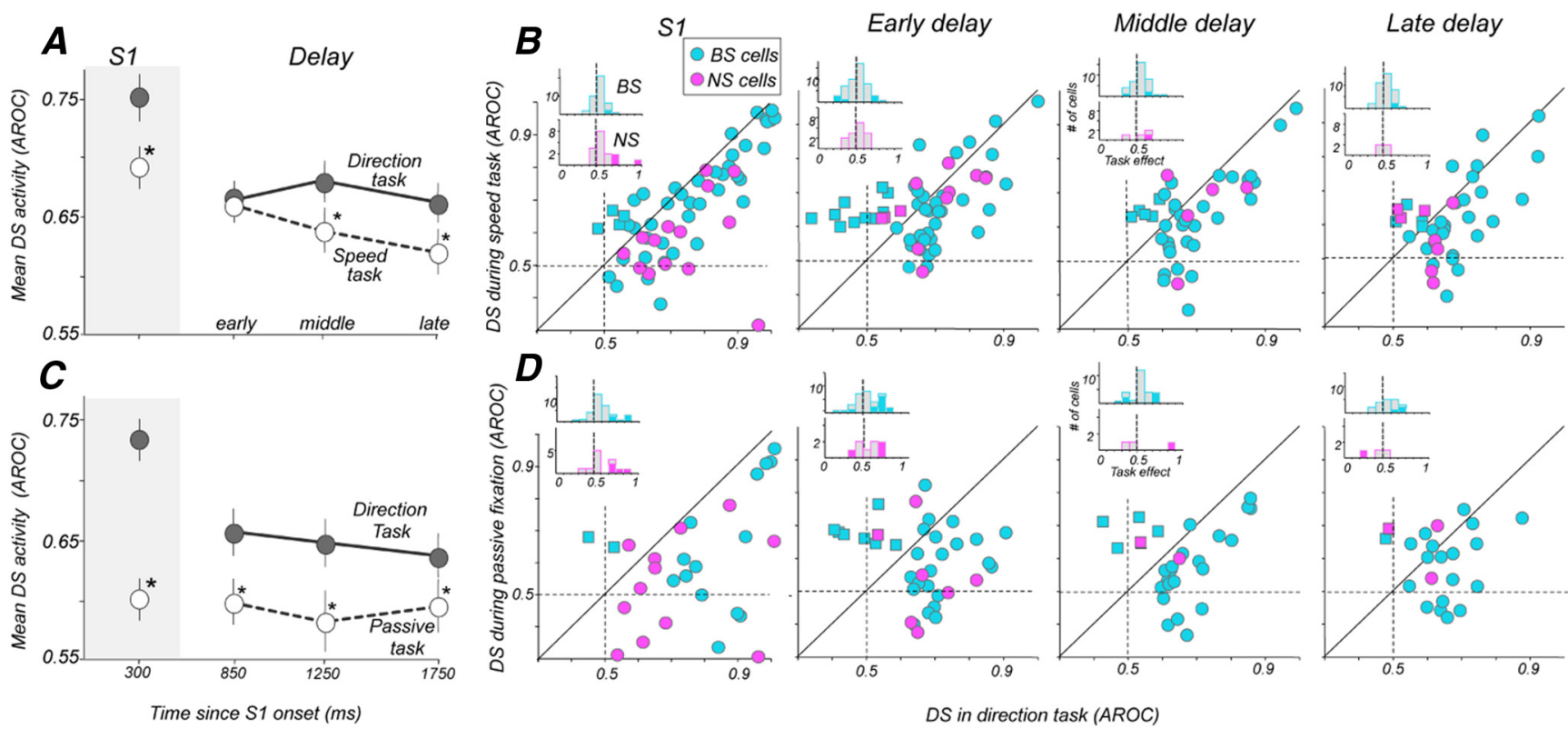

Figure 5. Direction-selective delay activity was affected by task demands. $A$, Average DS activity during direction and speed tasks recorded during three consecutive task periods: $S 1$ (200 - 400 $\mathrm{ms})$, early delay $(700-1000 \mathrm{~ms})$, middle delay (1000-1500 ms), and late delay $(1500-2000 \mathrm{~ms})$. For each epoch the data were calculated for all neurons with significant DS activity ( $p<0.05$, Wilcoxon sign rank test) in either task. Asterisk (*) indicates significant difference between tasks ( $p<0.05$, Wilcoxon sign rank test). $\boldsymbol{B}, \mathrm{DS}$ activity (AROC) of individual neurons during the direction and the speed discrimination tasks recorded during the early, middle, and late delays. All BS $(n=87)$ and NS ( $n=25)$ neurons were evaluated for significant DS activity in each task during each time period. Neurons with significant DS in the direction task (circles) and neurons with significant DS only during the speed task (squares) are shown separately. Insets in the upper left corner show distributions of task effects (TES) computed as [AROC $C_{\text {directon task }}-A R O C_{\text {non-direction task }}$ for all neurons. Neurons with significant TEs are shown by filled columns (bootstrap test, $\left.p<0.05\right)$. C, D, DS activity (AROC) during the direction and the passive fixation tasks. BS $(n=57)$ and NS cells $(n=16)$ were evaluated for DS activity during each time period. Conventions and labels are the same as in $\boldsymbol{A}$ and $\boldsymbol{B}$.

(Fig. 1A, middle panel). We were able to directly compare the behavior of 73 neurons (NS, $n=16$; BS, $n=57$ ) recorded during both the passive fixation and the direction discrimination tasks (Fig. 5C,D). Average DS activity recorded during S1 and the three delay periods in the two tasks, plotted in Figure $5 C$, shows that during passive fixation this activity was significantly weaker in all task epochs ( $\mathrm{S} 1, p=0.009$; early, $p=0.041$; middle, $p=0.042$; late, $p=0.049$, Wilcoxon sign-rank test). Under these conditions, in contrast to the speed task, the loss of DS activity for both cell types was similar ( $p=0.477$, Mann-Whitney $U$ test). The difference in DS on a cell-by-cell basis recorded during the two tasks is shown in Figure $5 D$.

The weakened DS delay activity of BS neurons during tasks not requiring retention of the preceding direction provides evidence for its role in sensory maintenance. Furthermore, these results further highlight the differential contribution of NS and BS neurons to different components of the sensory comparison task. While BS neurons showed only a small loss of DS in response to S1 when discriminating speeds (Hussar and Pasternak, 2009), during the maintenance phase of the same task these cells showed a significant loss of DS activity. On the other hand, delay activity became much less stimulus selective during the passive fixation task that did not require the animals to retain any information about S1. These results show that stimulus selectivity during the delay strongly depends on behavioral context, suggesting that it is likely to be used during the direction task. We should note that because of the small number of NS neurons with direction selective delay activity, these generalizations can only be applied to BS putative pyramidal neurons.

\section{Responses during S2 reflect remembered direction}

The data presented above showed that the information about the preceding stimulus was present in the form of transient distrib- uted signals, likely to be used during the direction discrimination task. Our analysis also revealed that BS, rather than NS, neurons were more likely to carry these signals, giving the putative pyramidal neurons a distinct role in the maintenance of sensory information. The difference between the cell types did not hold for activity recorded during the comparison stimulus (S2), since responses to S2 of both cell types were modulated by the S1 direction. We characterized these effects by comparing responses to identical S2 stimuli on trials when they were preceded by S1 moving in the same direction (S-trials) or by $\mathrm{S} 1$ moving in a different direction, offset by $90^{\circ}$ (D-trials), as shown in Figure $6 \mathrm{~A}$. The behavior of four example cells during S2, two NS (Fig. 6B, top row) and two BS (Fig. 6B, bottom row), illustrate the nature of response modulation we observed during the task: some cells showed stronger responses on S-trials (Fig. $6 \mathrm{~B}, \mathrm{~S}>\mathrm{D}$, left plots) and some cells showed preferences for D-trials (Fig. $6 \mathrm{~B}, \mathrm{D}>\mathrm{S}$, right plots). Since these effects were equally likely to occur in NS and BS cells (NS $=39 \%$; BS $=41 \%$ ), for further analysis the data from both groups are combined. In our analysis, for cells with excitatory responses to S2 $(n=66)$, higher firing rates on S-trials or D-trials were taken as an indication of a given cell's trial preference, while for cells with suppressive responses to S2 $(n=28)$, lower firing rates were indicative of the cell's trial preference. We used ROC analysis to quantify the differences in responses during the two types of trials. The results for all neurons are shown in Figure 6C. In this analysis, AROCs $>0.5$ indicate stronger responses on S-trials ( $>$ D, cooler colors), and values $<0.5$ indicate stronger responses on $\mathrm{D}$-trials $(\mathrm{D}>\mathrm{S}$, indicated by warmer colors). The values $\sim 0.5$ (the green shades) represent cells with no reliable difference between responses during the two trial types $(S=D)$. For display purposes the cells were grouped and sorted by the onset time of the effect (see Materials and Methods). An important feature of these results is that each type of effect, S > D and 

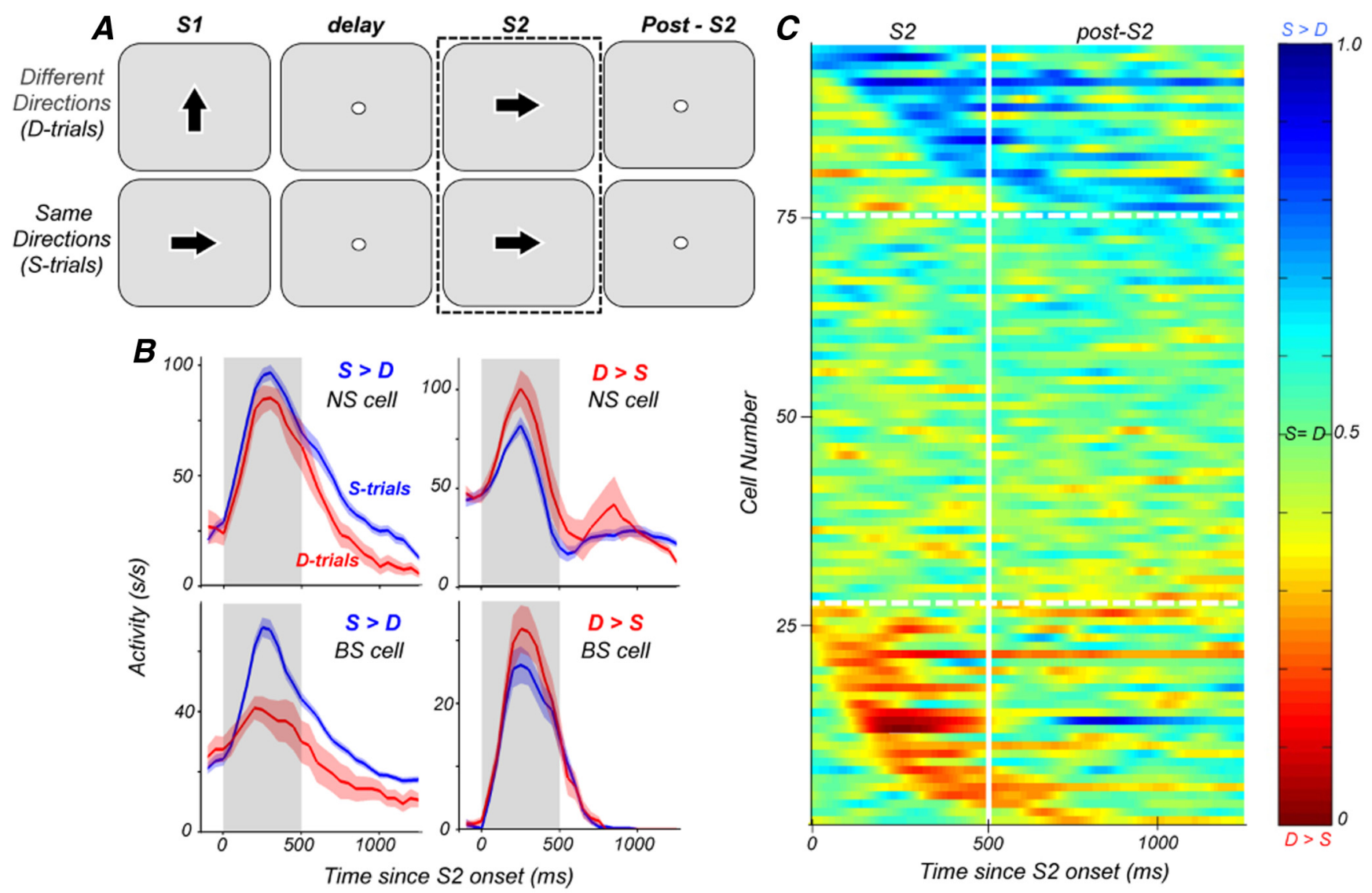

Figure 6. Comparison effects (CE) during S2.A, Diagram illustrating the two types of trials used to evaluate CE, S-trials (same), and D-trials (different). $\boldsymbol{B}$, Example responses during S2 of two NS (top row) and two BS (bottom row) neurons showing the two types of modulation by $S 1$ direction. The two neurons on the left fired more on $S$-trials $(S>D$, blue lines), while the two neurons on the right responded more on D-trials ( $D>S$, red lines). Note that both NS and BS cells showed such effects. C, CE in all neurons quantified with ROC analysis (BS, $n=76 ; \mathrm{NS}, n=18)$. AROC values $>$ 0.5 (cooler colors) signify higher activity on S-trials, and AROC values $<0.5$ (warmer colors) signify stronger activity on D-trials. Neurons were sorted by timing and sign of their comparison effects. Note, $S$ and $D$ trials are signaled by different groups of cells, $S>D$ (blue) and $D>S$ (red).

$\mathrm{D}>\mathrm{S}$, is carried by a distinct group of neurons. To further characterize the modulation of S2 responses by the preceding stimulus, all neurons were assigned into three subgroups on the basis of their ROC values: $\mathrm{S}>\mathrm{D}(\mathrm{AROC} \geq 0.65 ; n=20,21 \%), \mathrm{D}>\mathrm{S}$ (AROC $\leq 0.35 ; n=26,28 \%)$ and $\mathrm{S}=\mathrm{D}(n=48,51 \%)$.

To directly compare the two types of effects (i.e., $S>D$ and $\mathrm{D}>\mathrm{S}$ ), we converted AROCs in such a way that a value $>0.5$ represented a greater response during that neuron's preferred trial type (either S-trials or D-trials). These data, shown in Figure $7, A-C$, illustrate both similarities and differences between $S>D$ and D $>$ S effects, which we will term "comparison" effects. Both effects were of similar strength and extended past the offset of the S2. However, the effect carried by the D $>$ S group emerged significantly earlier (D > S, $250 \mathrm{~ms} \pm 34 ; \mathrm{S}>\mathrm{D}, 390 \mathrm{~ms} \pm 49$; $p=$ 0.02; Mann-Whitney $U$ test), reaching its peak at $340 \mathrm{~ms}$ after S2 onset. Cells in the $S>D$ group reached their peak on average 300 ms later (Fig. 7B). These differences in onset times between the two types of effects cannot be attributed to differences in the time course of their stimulus responses, since the two groups had similar latencies to $\mathrm{S} 2(\mathrm{~S}>\mathrm{D}, 100 \mathrm{~ms} \pm 36$; $\mathrm{D}>\mathrm{S}$ cells, $130 \mathrm{~ms} \pm 24$; $p=0.32$; Mann-Whitney $U$ test). While both types of signals persisted after the offset of $\mathrm{S} 2$, the $\mathrm{S}>\mathrm{D}$ effects persisted longer (Fig. 7A; $p<0.05$; Mann-Whitney $U$ test). Because in a small subset of cells with $S>D$ effects emerged at different times after the offset of S2 (see Fig. 6C), the average AROC curve for the $\mathrm{S}>\mathrm{D}$ group remained elevated throughout the post-S2 period until the monkeys reported their decision.
The above analysis was limited to responses recorded on trials with the largest direction difference $\left(90^{\circ}\right)$ easily discriminable by both animals. However, during each recording session the animals were presented with a range of direction differences and their performance decreased as the two directions became more similar (see psychometric functions in Fig. 1B). We examined whether the magnitude of comparison effects paralleled behavioral performance and decreased as the two directions became more similar. This type of effect would provide compelling evidence in support of response modulations during S2 representing the sensory comparison. We tested this hypothesis by calculating comparison effects on trials with smaller differences in directions. The AROC calculated from trials of the maximal difference in direction $\left(90^{\circ}\right)$ was then subtracted from the AROC calculated for smaller differences in direction. (Fig. 7C). In this metric, negative values indicate a decrease in the comparison effect at smaller differences in direction between S1 and S2. We found a significant relationship between the size of the comparison effect and the difference in direction, with activity during both types of trials becoming more similar with smaller differences in direction $(p<$ $0.001, r^{2}=0.419$, Pearson's correlation). This relationship held for both $\mathrm{S}>\mathrm{D}\left(p<0.001, r^{2}=0.754\right.$, Pearson's correlation $)$ and $\mathrm{D}>\mathrm{S}\left(p<0.001, r^{2}=0.613\right.$, Pearson's correlation) cells. This scaling of the comparison effect with direction difference between $\mathrm{S} 1$ and $\mathrm{S} 2$ was similar in the two monkeys (Fig. 7C; Monkey 1 , solid line; Monkey 2, broken line, ANOVA, $p=0.73$ ). However, the more accurate Monkey 2 had a significantly larger pro- 
portion of cells carrying comparison effects (Fig. 7D; 200-500 ms, $\chi^{2}$ test, $p=$ $0.03)$. In addition to the lower incidence of cells with comparison effects, the less accurate animal also showed somewhat lower AROC values indicative of weaker comparison effects, although this difference did not reach significance (Fig. 7E; Monkey $1, n=6$ Monkey 2, $n=20$; $p=$ 0.13; Mann-Whitney $U$ test). While this correlation between the incidence of cells with comparison effects and performance is compelling, it cannot be generalized since it is based on the comparison between two monkeys. Together, these results suggest that the two types of trials characteristic of our task were signaled by distinct neurons and that these signals become smaller when the direction differences become smaller, suggesting that the observed modulation reflected the process of sensory comparison. The behavioral relevance of these signals is underscored by the correlation between the lower performance and weaker comparison effects. As we will show below, the close relationship between the comparison effects and behavior was also revealed by the analysis of activity associated with behavioral report.

To further examine the behavioral utility of comparison effects and whether these effects occur when animals are not actively engaged in the discrimination task, we compared activity in a subset of neurons during the direction task and the passive fixation task (Fig. 1A, middle panel). This analysis was performed on a small subset of neurons with comparison effects $(n=14)$ and a sufficient number of trials in both tasks. Because we had relatively a small number of neurons with a sufficient number of trials recorded in both tasks, and cells with $\mathrm{S}>\mathrm{D}$ and $\mathrm{D}>\mathrm{S}$ modulation showed similar task effects, their data were combined. Figure $8 \mathrm{~A}$ shows the relative response of an example neuron on $\mathrm{S}$ - and D-trials during the two tasks. This neuron showed a robust comparison effect during the direction task (left plot) that decreased substantially during the passive fixation task. The other PFC neurons also exhibited strong attenuation of the average comparison effect during passive fixation, as shown by the average comparison effects and the scatter plots for the neurons studied under the two behavioral conditions (Fig. $8 B, C$ ). The data show that when monkeys were not required to actively engage in direction discrimination, comparison-related activity drastically decreased ( $p=0.008$, Wilcoxon sign-ranked test). This result demonstrates that response modulation in the PFC recorded during the comparison phase of the discrimination task occurs largely when animals are actively engaged in sensory comparisons.

While stimulus-selective delay activity was transient and did not show a consistent representation of S1 direction, its decrease during tasks not requiring direction discrimination suggests that this activity may be used. Since its utilization would be most advantageous during the comparison phase of the task, we asked whether the comparison effects identified in individual neurons depended on these cells also showing DS activity during the preceding delay. While $59 \%(n=27)$ of cells with comparison effects exhibited DS activity at any time during the delay, $41 \%(n=19)$ showed no significant DS delay activity at all. This result suggests that in individual neurons, comparison effects do not necessarily require their own stimulus-selective delay activity and are more likely to rely on the information distributed among many neurons.

\section{Comparison effects correlate with behavioral choice}

The presence of signals reflecting similarities and differences between $S 1$ and S2 raises the question whether these signals are used in the decision process. We addressed this question by first determining whether the activity that followed the onset of S2 was predictive of the behavioral report (choice probability, CP) and then examined the relationship between $\mathrm{CP}$ and the comparison effects.

We began by calculating CP (Britten et al., 1996; Zaksas and Pasternak, 2006) for all PFC neurons with a sufficient number of trials $(n=84)$. This analysis was applied only to activity recorded on S-trials when S1 and S2 moved in the same direction in neurons with sufficient numbers of "same" and "different" reports (see Materials and Methods). The analysis revealed that more than half of the PFC neurons showed decision-related activity and identified two distinct groups of neurons associated with the behavioral report: neurons that fired more before "same" reports 


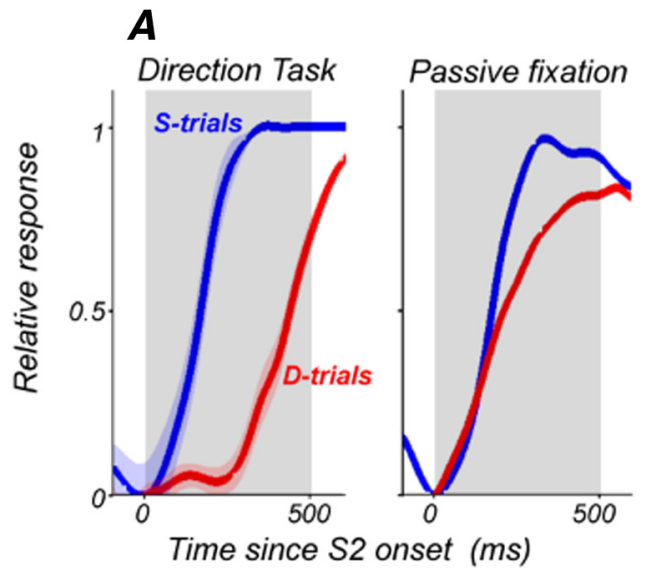

B
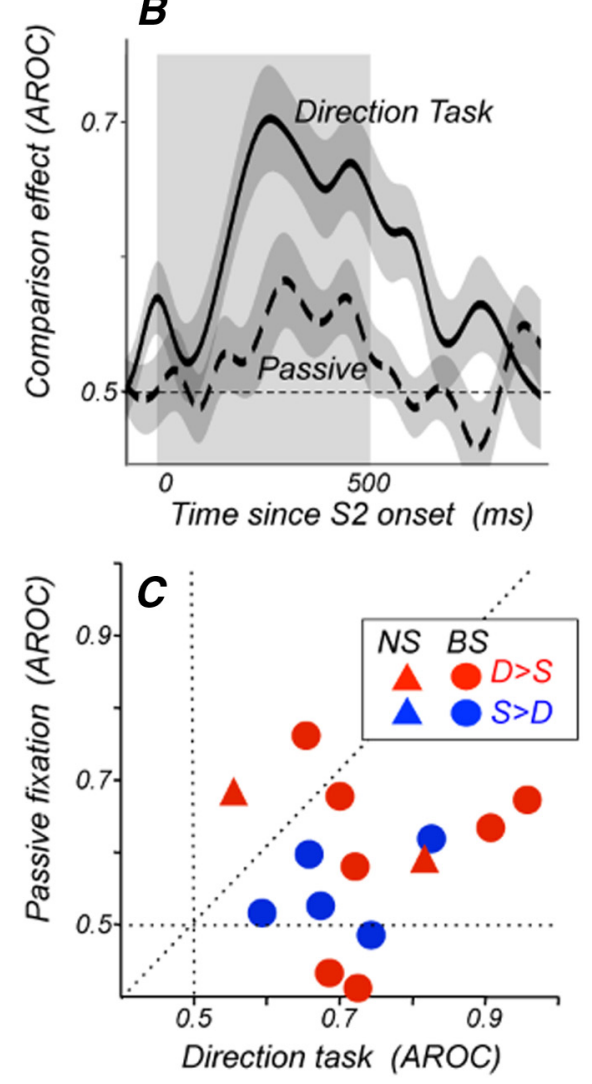

Figure 8. Attenuation of comparison effects during passive fixation. $A$, Relative responses to S2 on S- and D-trials of an example neuron recorded during the direction (left) and passive fixation (right) tasks. $\boldsymbol{B}$, Average comparison effects recorded during the two tasks (NS $=2$, $B S=12)$. C, Cell-by-cell comparison effects measured during the direction task and passive fixation. The data represent activity recorded during $100-300 \mathrm{~ms}$ after the onset of $\mathrm{S2}$. Comparison effects were weaker during the passive task (Wilcoxon sign-rank test, $p=0.004$ ).

(right button, 26\%, $n=22$ ), and neurons that fired more before "different" reports (left button, $31 \%, n=26$ ). The remaining neurons $(43 \%, n=36)$ showed no differential decision-related activity. Figure $9 A$ shows the average $C P$ for neurons with higher activity before "same" (blue line) and "different" reports (red line) during S2 and post-S2 periods. CPs of cells associated with "different" reports reached their maximum during the S2, while CPs of cells signaling "same" reports reached their maximum later, after the offset of S2. Figure $9 B$ shows the distributions of CPs contributing to each curve during three time points following the onset of S2. These distributions show that activity signal-
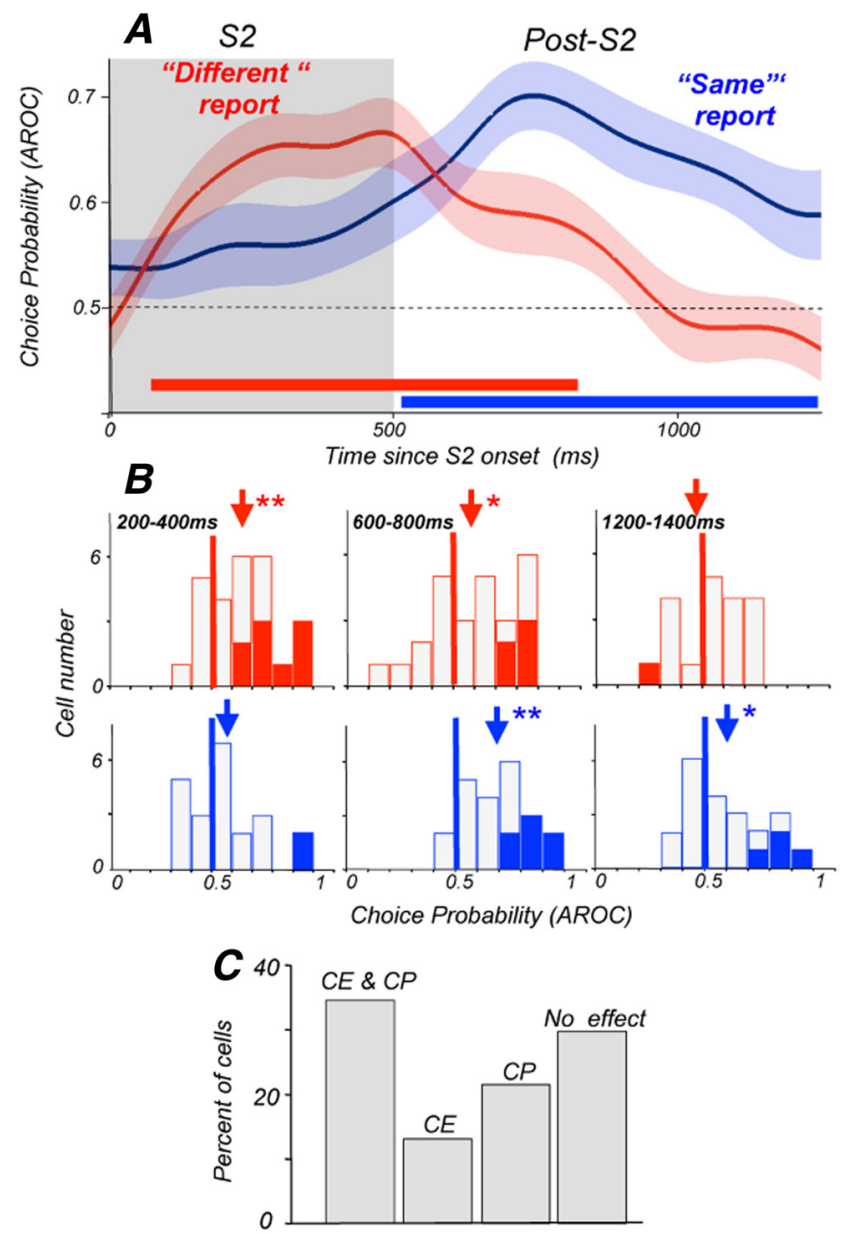

Figure 9. Activity during $\$ 2$ predicts perceptual report. $A$, Choice probability of neurons more active before "different" $(n=24)$ and before "same" $(n=17)$ reports. Thick colored lines along the $x$-axis indicate period of significance (Mann-Whitney $U$ test, $p<0.05$ ). Shadings represent \pm SEM. $\boldsymbol{B}$, Distributions of CPs for neurons contributing to CP curves shown in $\boldsymbol{A}$. Distribution of CPs during $200-400 \mathrm{~ms}, 600-800 \mathrm{~ms}$, and $1200-1400 \mathrm{~ms}$ after the onset of S2. Arrows point to mean CPs for each period; ${ }^{*} p<0.05 ;{ }^{* *} p<0.01$. C, Incidence of $C E$ and $C P$ signals co-occurring in the same neurons ( $C P$ and $C E, n=29 ; C$ only, $n=11 ;(P$ only, $n=18$; no effect, $n=25$ ).

ing "different" reports (shown in red) became significant early in the response $(200-400 \mathrm{~ms}, \mathrm{CP}=0.65, p=0.0002 ; 600-800 \mathrm{~ms}$, $\mathrm{CP}=0.59, p=3.6 \times 10^{-4} ; 1200-1400 \mathrm{~ms}, \mathrm{CP}=0.46, p=0.47$; Mann-Whitney $U$ test) and disappeared about $500 \mathrm{~ms}$ before the response. The distributions of CP for cells associated with "same" reports (shown in blue) became significant later, reaching their maximum during the post-S2 period and largely persisting into the period of behavioral report $(200-400 \mathrm{~ms}, \mathrm{CP}=0.56 ; p=$ $0.15 ; 600-800 \mathrm{~ms}, \mathrm{CP}=0.69, p=1.2 \times 10^{-4} ; 1200-1400 \mathrm{~ms}$, $\mathrm{CP}=0.60, p=0.045$; Mann-Whitney $U$ test). Overall, these data show that decision-related activity appeared shortly after the onset of S2 and had a distinct time course for each of the two types of reports, appearing shortly after the onset of the comparison stimulus and persisting until the behavioral report was made.

The two groups of neurons with complementary decision-related activity paralleled the two groups of neurons identified as carrying comparison effects (CEs) (see Fig. 7). This similarity prompted us to examine whether the two types of activity co-occur in the same neurons. This analysis, summarized in Figure $9 C$, revealed that $35 \%(n=29)$ of the PFC neurons carried both choice and comparison-related activity during S2 and/or post-S2 periods 
(CP \& CE), 22\% ( $n=18)$ showed only choice-related activity (CP), and the minority of cells, $13 \%(n=11)$, exhibited comparison effects but no choice-related signals (CE). We examined the relationship between the two types of activity co-occurring in the same neurons. Figure $10 \mathrm{~A}$ plots the average $\mathrm{CE}$ (solid line) and CP (broken line) computed for the same neurons. The data for $\mathrm{S}>\mathrm{D}$ cells (blue lines, $n=13$ ) and $\mathrm{D}>\mathrm{S}$ cells (red lines, $n=16$ ) are plotted separately. Although both groups showed average $\mathrm{CPs}$ comparable in strength to their CEs, choice-related activity reached its peak consistently later than did the comparison effects. Figure $10 \mathrm{~B}$ compares the times of maximal effects for these two signals on a cell-by-cell basis. $\mathrm{CPs}$ for $\mathrm{S}>\mathrm{D}$ neurons reached their average maximal effect $190 \mathrm{~ms}$ later than the maximal comparison effects (Fig. $10 C, p=0.012$, Wilcoxon Sign-Rank task). Similarly, D $>$ S neurons showed their peak CP $145 \mathrm{~ms}$ later than their maximal comparison effects (Fig. 10C, $p=0.006$, Wilcoxon Sign-Rank task).

We also examined the strength and sign of these two types of signals within individual neurons. For this analysis we included all neurons carrying comparison effects during or after S2. We found a strong correlation between the strength of $\mathrm{CE}$ and $\mathrm{CP}$ (Fig. 10D-F). Neurons preferring $\mathrm{S}$-trials $(\mathrm{S}>\mathrm{D})$ also tended to fire more before "same" reports, while cells that preferred D-trials $(\mathrm{D}>\mathrm{S})$ also showed higher rates before "different" reports. A positive correlation between these two effects was observed during S2 (Fig. $10 D, \mathrm{~S}>\mathrm{D}, p=0.009, r^{2}=0.61$; $\mathrm{D}>\mathrm{S}, p=0.002, r^{2}=0.58$, Pearson's correlation) and during the early post-S2 period (Fig. $10 E$; $\mathrm{S}>\mathrm{D}, p=1.2 \times 10^{-4}$, $r^{2}=0.79 ; \mathrm{D}>\mathrm{S}: p=0.03, r^{2}=0.44$, Pearson's correlation). In contrast, ROC values of cells showing no preference for trial type $(S=D)$ showed no significant correlation with CP (Fig. $10 F ; p=0.978$; $\left.r^{2}=0.004\right)$. This relationship supports the hypothesis that the PFC neurons with more reliable comparison effects are more strongly related to the animal's perceptual report. Overall, the difference in the timing between the two types of effects, and the strong correlation between them, is consistent with the possibility that the information about similarities and differences between stimuli is likely to be used by decision-related circuitry.

\section{Discussion}

Our results demonstrate distinct contributions of BS and NS neurons to anticipatory, maintenance, and decision-related processes in the PFC. While both cell types showed equivalent representations of direction in response to behaviorally relevant visual motion, during the delay BS neurons were more active and more likely to exhibit anticipatory and stimulus-selective activity
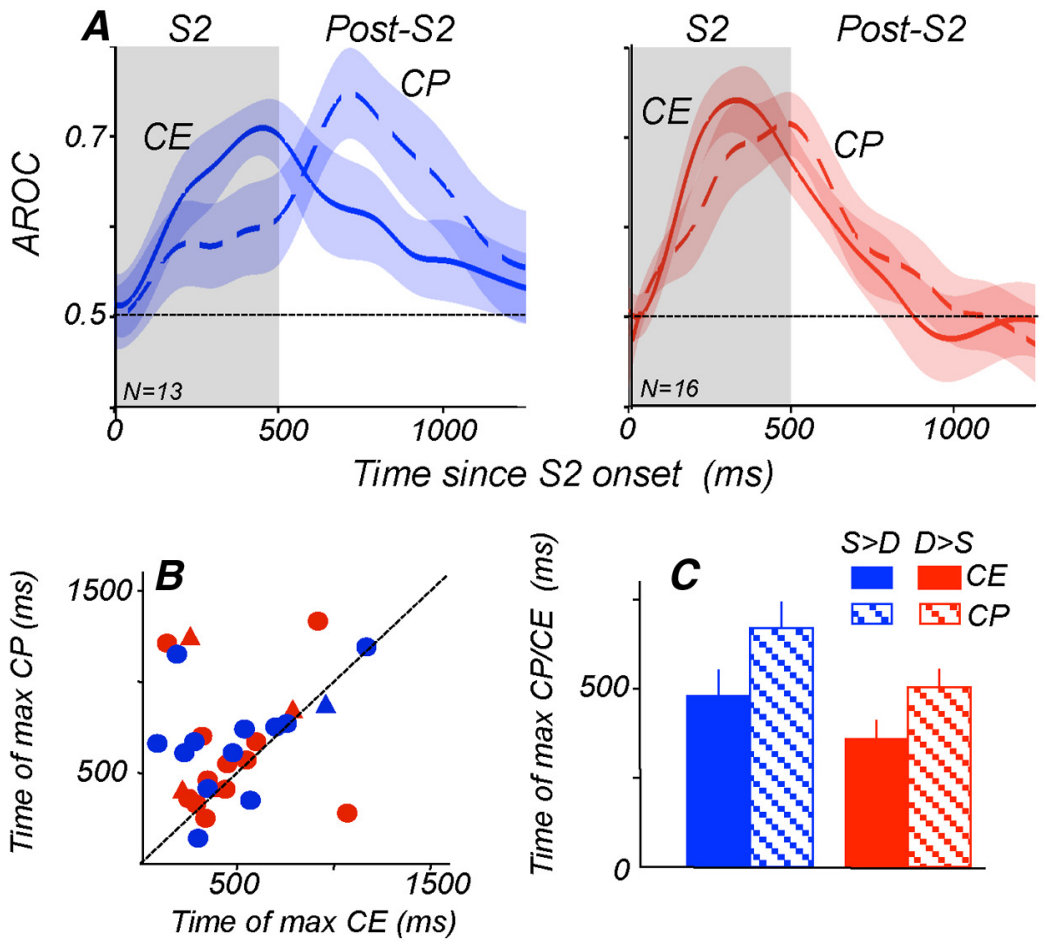

Comparison effects (CEs) and choice-related (CP) signals. $A$, Time course of CE and CP in $S>$ D neurons $(n=13$, blue Average times of maximal CP and CE. CE preceded CP by $145 \mathrm{~ms}$ in D $>$ S cells ( $p=0.006$, Wilcoxon sign-rank test) and by $190 \mathrm{~ms}$ in $S>D$ cells $(p=0.012$, Wilcoxon sign-rank test). $D-E$, Correlation between CE and C $P$ for individual $S>D$ and $D>S$ cells a $200-400 \mathrm{~ms}(\boldsymbol{D}, S>D, p=0.009 ; D>S, p=0.002)$ and at $600-800 \mathrm{~ms}(\boldsymbol{E}, S>D, p=1.2 \times 10-4 ; D>S, p=0.03)$ after S2 onset. $\boldsymbol{F}$, Neurons with no $C E(S=D)$ showed no correlation between $C E$ and $C P(p=0.979)$.

than NS neurons, suggesting a unique role for these neurons in maintaining task-related activity in the absence of sensory stimulation. However, during S2 both cell types again exhibited comparable levels of task-related activity, showing responses that were modulated by the preceding direction. This modulation scaled with the difference between the two stimuli and was strongly correlated with activity predicting the subsequent choice.

\section{Cell classification}

In our analysis we used waveform durations to distinguish between putative pyramidal cells and putative inhibitory interneurons. The presence of a bimodal distribution of waveform durations in our dataset (see Hussar and Pasternak, 2009, their Fig. $1 H$ ) provided the basis for this distinction that was also supported by characteristic differences in their baseline and response firing rates (Hussar and Pasternak, 2009). We should note 


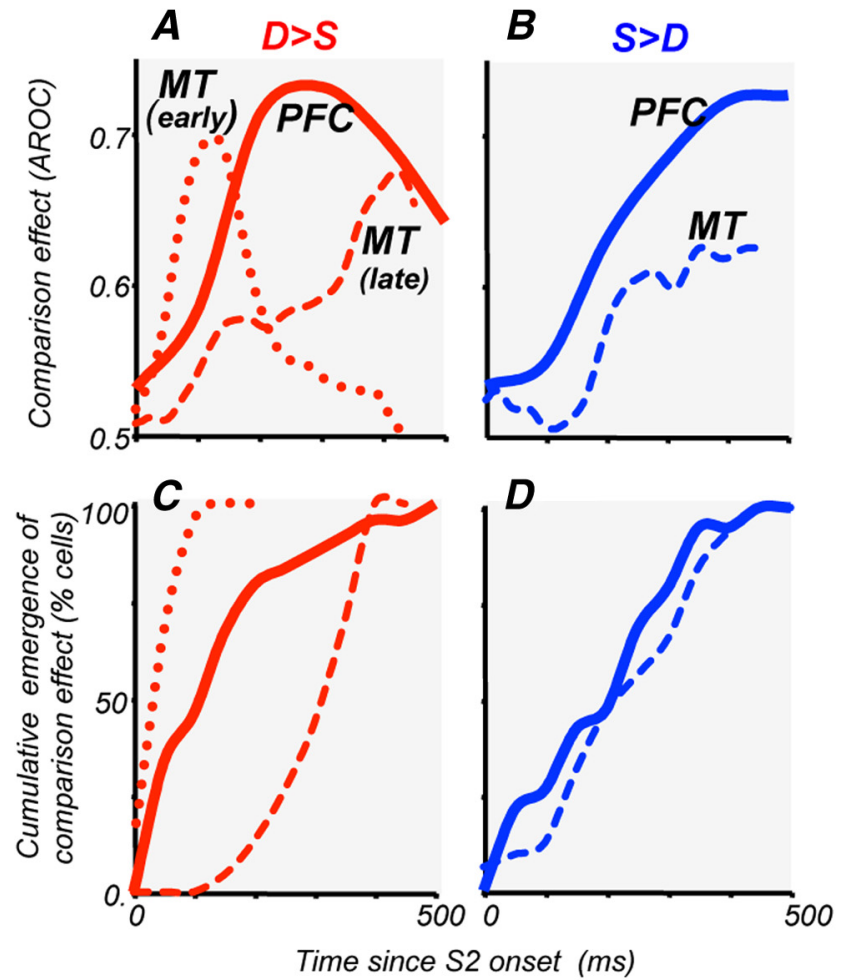

E

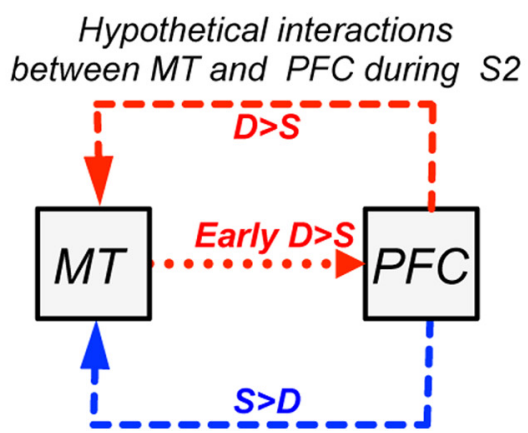

Figure 11. Comparison effects in PFC and MT. Comparison effects during $S 2$ recorded in the PFC and in MT. MT data are replotted from Lui and Pasternak (2011). $A$, CE for D > S cells in PFC (solid line) and two subpopulations of $D>S$ cells in MT, early (dotted line) and late (broken line) identified in MT. B, CE for S > D cells in PFC (solid line) and MT (broken line). C, D, Cumulative emergence of comparison effects in MT and PFC. Conventions and labels are identical to $A$ and $B$. E, Hypothetical interactions between MT and PFC during S2.

that this classification ignores a number of complexities associated with the multitude of neuronal subtypes among interneurons (Markram et al., 2004) and with dependence of waveform duration on the size and conduction velocity of pyramidal neurons, which can vary with cortical areas (Vigneswaran et al., 2011). Nevertheless, it provided a very useful tool, and the two types of neurons in the PFC and other cortical areas, classified with this approach, have been shown to play distinct roles in a range of behavioral tasks (Mitchell et al., 2007; Diester and Nieder, 2008; Hussar and Pasternak, 2009; Johnston et al., 2009).

\section{Stimulus selective activity during the delay}

We found that BS neurons were more likely than NS cells to represent stimulus direction during the delay. This unique role for BS cells in maintaining stimulus representation provides some support for predictions made by computational models of delay activity (Wang, 2001; Engel and Wang, 2011).
It is often assumed that during working memory tasks individual neurons maintain persistent signals representing the remembered stimulus across multiple seconds of delay (Durstewitz et al., 2000; Brody et al., 2003) (but see (Shafi et al., 2007). Our data provide evidence against this view. We found that periods of DS activity were relatively brief, rarely exceeding $400 \mathrm{~ms}$, often changed sign, and were unrelated to response selectivity during S1. Several studies have provided compelling examples of individual neurons with persistent stimulus-specific delay activity but focused their analysis largely on stimulus representations in the population, either by documenting the incidence of neurons with stimulus-selective signals encountered throughout the delay (Romo and Salinas, 2003) or by showing averaged delay selectivity (Miller et al., 1996). In our data we also see a continuous representation of direction within the population activity. However, our analysis highlights that DS activity in individual neurons was transient and inconsistent, a feature detected largely because we compared directly stimulus preferences during S1 and the delay, keeping track of their signs. Indeed, a closer inspection of stimulus selectivity of individual neurons for entire PFC datasets shown in other studies (Wallis and Miller, 2003; Shafi et al., 2007; Jun et al., 2010; Cromer et al., 2011) also reveals the transient nature of this selectivity, similar to that reported here.

The lack of consistency in stimulus selectivity between S1 and delay extended throughout the trial, since there was no consistent relationship between comparison effects and the preceding delay activity. Other studies noted a similar lack of continuity in selectivity across trials (Fuster, 1973; Miller et al., 1996). Overall, the transient nature of DS activity during the delay and its variation within individual cells suggests that the preceding stimulus is more likely to be dynamically represented in the population. Two recent studies focused on the dynamic and transient nature of stimulus selectivity during the delay and successfully used population-based approaches to decoding stimulus identity (Meyers et al., 2008; Barak et al., 2010).

\section{PFC cells represent similarities and differences between comparison stimuli}

Many neurons carried a comparison effect, representing the difference between S1 and S2. These comparison effects were equally likely to be carried by BS and NS neurons despite the absence of stimulus-selective activity in late delay in the latter cell type, further supporting the notion that these effects were unlikely to be the result of stimulus-selective delay activity "bleeding in" to the test response (Engel and Wang, 2011). This apparent independence between comparison effects and delay activity raises an obvious question. How do neurons access the information about the preceding stimulus? Although our results do not provide an unequivocal answer to this important question, the comparison between response modulation during S2 in the PFC and MT during similar tasks suggests one potential partial solution (Fig. 11).

While in the PFC "same" and "different" trials are represented by roughly equivalent numbers of PFC neurons (Miller et al., 1996; Freedman et al., 2002), neurons with weaker responses on "same" trials (also termed match suppression) appear to be more prevalent in sensory areas contributing feedforward inputs to the PFC (Miller et al., 1991, 1996; Lui and Pasternak, 2011). Although the interactions between neurons carrying comparison effects at both levels have not been studied directly, recent computational models incorporated interactions between PFC and sensory neurons as a mechanism by which comparison effects might arise (Ardid et al., 2007; Engel and Wang, 2011). Particu- 
larly relevant here is the work of Engel and Wang (2011) who proposed a specific comparison network arising from differential influences of bottom-up and top-down activation. While their model was designed to explain match-to-sample tasks and assumes persistent firing during the delay, our data support some of its predictions, including scaling of comparison effects with direction difference and the reliance of decision circuits on the activity of cells representing comparison effects. Our data provide additional constrains on this and other models in the form of timing differences between the two types of comparison effects.

One of the striking features of the observed comparison effects was their different time course, with activity signaling D-trials emerging earlier than the activity signaling S-trials. One explanation for the earlier occurrence of $\mathrm{D}>\mathrm{S}$ effect may lie in potentially different origins of the two types of effects. We recently identified distinct groups of MT cells representing S- and D-trials during a similar discrimination task (Lui and Pasternak, 2011). These effects were characterized by the late emergence of $S>D$ effects and by the presence of a subgroup of cells with early D $>S$ effects. In that paper we hypothesized that the early D $>$ S effects have local origins within MT, while the late $\mathrm{D}>\mathrm{S}$ and the $\mathrm{S}>\mathrm{D}$ effects may have top-down origins, possibly from the PFC. Figure 11 illustrates the differences in the time course of these effects in the two brain areas, suggestive of a feedforward relationship between early D $>$ S activity in MT and the trailing D $>$ S effect in the PFC, coupled with PFC sending signals to MT in the form of later occurring $\mathrm{D}>\mathrm{S}$ and $\mathrm{S}>\mathrm{D}$ activity. According to this scenario (Fig. $11 E$ ), the process of sensory comparisons originates in MT with the appearance of early D $>$ S effects. These signals are read out in the PFC and incorporated into the comparison process, which gives rise to the top-down $\mathrm{S}>\mathrm{D}$ and $\mathrm{D}>\mathrm{S}$ effects that, in turn, are provided to MT. This interpretation is supported by the absence of the early D $>$ S component in MT responses under task conditions in which comparison effects in MT could only reflect top-down influences (Lui and Pasternak, 2011).

\section{Comparison effects and perceptual decision}

The observed comparison effects showed a strong link with behavior, both across and within individual animals. This could be seen in a greater incidence of comparison effects in the better performing animal, in their near absence during passive fixation, and in their strong positive correlation with decision-related activity, suggesting that the comparison effects are likely to be used in perceptual decision. The observation that in individual neurons CPs trailed comparison effects by $\sim 100 \mathrm{~ms}$ (Fig. $10 \mathrm{~B}$ ) is consistent with this possibility.

As with comparison effects, we found a difference in the time course between the two types of choice-related effects, with activity that preceded "different" reports emerging and terminating earlier than "same" reports. This difference could be related to motor preparation, since in our task the choice and the motor response were linked, and in both monkeys the data were collected from the left hemisphere. However, this is unlikely, because activity associated with motor preparation has been shown to be similar in several cortical areas across hemispheres (Wallis and Miller, 2003; Hernandez, 2007). A more likely explanation of the later onset of "same" report is its hypothesized dependence on the $S>D$ comparison effect, which emerged relatively late in the response.

\section{Functional specialization of NS and BS neurons during motion comparison task}

Our results provide the first demonstration of distinct contributions of the two cell types to task-related activity during sensory comparisons. In the presence of visual motion, both cell types showed direction selective responses and comparison effects, reflecting both the bottom-up influences and local transformations. While our data do not provide insights into whether these responses emerged independently in the two cell classes or were a product of interactions between them (Constantinidis and Goldman-Rakic, 2002), they show that both groups are involved in representing sensory information. However, in the absence of sensory stimulation, neural activity was dominated by BS neurons, which signaled the elapsed time and carried transient and transformed representations of the remembered direction. Because these neurons are a likely source of top-down projections arising in the PFC, this result elucidates the nature of its influences on other cortical neurons. Our results also shed light on the flexibility of this apparent functional specialization; both cell groups were sensitive to the behavioral context but during different portions of the trial: NS cells were more likely to signal the change in task demands during sensory stimulation, while BS cells were more sensitive to the behavioral context during the delay. These results set important constraints on models of sensory comparison networks and sensory working memory.

\section{References}

Ardid S, Wang XJ, Compte A (2007) An integrated microcircuit model of attentional processing in the neocortex. J Neurosci 27:8486-8495.

Barak O, Tsodyks M, Romo R (2010) Neuronal population coding of parametric working memory. J Neurosci 30:9424-9430.

Barbas H (1988) Anatomic organization of basoventral and mediodorsal visual recipient prefrontal regions in the rhesus monkey. J Comp Neurol 276:313-342.

Britten KH, Shadlen MN, Newsome WT, Movshon JA (1992) The analysis of visual motion: a comparison of neuronal and psychophysical performance. J Neurosci 12:4745-4765.

Britten KH, Newsome WT, Shadlen MN, Celebrini S, Movshon JA (1996) A relationship between behavioral choice and the visual responses of neurons in macaque MT. Vis Neurosci 13:87-100.

Brody CD, Romo R, Kepecs A (2003) Basic mechanisms for graded persistent activity: discrete attractors, continuous attractors, and dynamic representations. Curr Opin Neurobiol 13:204-211.

Connors BW, Gutnick MJ (1990) Intrinsic firing patterns of diverse neocortical neurons. Trends Neurosci 13:99-104.

Constantinidis C, Goldman-Rakic PS (2002) Correlated discharges among putative pyramidal neurons and interneurons in the primate prefrontal cortex. J Neurophysiol 88:3487-3497.

Contreras D, Palmer L (2003) Response to contrast of electrophysiologically defined cell classes in primary visual cortex. J Neurosci 23:6936-6945.

Cromer JA, Roy JE, Buschman TJ, Miller EK (2011) Comparison of primate prefrontal and premotor cortex neuronal activity during visual categorization. J Cogn Neurosci 23:3355-3365.

Diester I, Nieder A (2008) Complementary Contributions of prefrontal neuron classes in abstract numerical categorization. J Neurosci 28:7737-7747.

Durstewitz D, Seamans JK, Sejnowski TJ (2000) Neurocomputational models of working memory. Nat Neurosci 3:1184-1191.

Engel TA, Wang XJ (2011) Same or different? A neural circuit mechanism of similarity-based pattern match decision making. J Neurosci 31:69826996.

Freedman DJ, Riesenhuber M, Poggio T, Miller EK (2002) Visual categorization and the primate prefrontal cortex: neurophysiology and behavior. J Neurophysiol 88:929-941.

Fuster JM (1973) Unit activity in prefrontal cortex during delayed-response performance: neuronal correlates of transient memory. J Neurophysiol 36:61-78.

Gray CM, McCormick DA (1996) Chattering cells: superficial pyramidal 
neurons contributing to the generation of synchronous oscillations in the visual cortex. Science 274:109-113.

Hartigan JA, Hartigan PM (1985) The dip test of unimodalit. Ann Statist 13:70-84.

Hernández A, Nácher V, Luna R, Zainos A, Lemus L, Alvarez M, Vázquez Y, Camarillo L, Romo R (2010) Decoding a perceptual decision process across cortex. Neuron 66:300-314.

Hussar C, Pasternak T (2010) Trial-to-trial variability of the prefrontal neurons reveals the nature of their engagement in a motion discrimination task. Proc Natl Acad Sci U S A 107:21842-21847.

Hussar CR, Pasternak T (2009) Flexibility of sensory representations in prefrontal cortex depends on cell type. Neuron 64:730-743.

Johnston K, DeSouza JF, Everling S (2009) Monkey prefrontal cortical pyramidal and putative interneurons exhibit differential patterns of activity between prosaccade and antisaccade tasks. J Neurosci 29:5516-5524.

Jun JK, Miller P, Hernández A, Zainos A, Lemus L, Brody CD, Romo R (2010) Heterogenous population coding of a short-term memory and decision task. J Neurosci 30:916-929.

Lui LL, Pasternak T (2011) Representation of comparison signals in cortical area MT during a delayed direction discrimination task. J Neurophysiol 106:1260-1273.

Markram H, Toledo-Rodriguez M, Wang Y, Gupta A, Silberberg G, Wu C (2004) Interneurons of the neocortical inhibitory system. Nat Rev Neurosci 5:793-807.

McCormick DA, Connors BW, Lighthall JW, Prince DA (1985) Comparative electrophysiology of pyramidal and sparsely spiny stellate neurons of the neocortex. J Neurophysiol 54:782-806.

Meyers EM, Freedman DJ, Kreiman G, Miller EK, Poggio T (2008) Dynamic population coding of category information in inferior temporal and prefrontal cortex. J Neurophysiol 100:1407-1419.

Miller EK, Cohen JD (2001) An integrative theory of prefrontal cortex function. Annu Rev Neurosci 24:167-202.

Miller EK, Erickson CA, Desimone R (1996) Neural mechanisms of visual working memory in prefrontal cortex of the macaque. J Neurosci 16:5154-5167.

Miller EK, Li L, Desimone R (1991) A neural mechanism for working and recognition memory in inferior temporal cortex. Science 254:1377-1379.

Mitchell JF, Sundberg KA, Reynolds JH (2007) Differential attentiondependent response modulation across cell classes in macaque visual area V4. Neuron 55:131-141.

Nowak LG, Azouz R, Sanchez-Vives MV, Gray CM, McCormick DA (2003) Electrophysiological classes of cat primary visual cortical neurons in vivo as revealed by quantitative analyses. J Neurophysiol 89:1541-1566.

Petrides M (2005) Lateral prefrontal cortex: architectonic and functional organization. Philos Trans R Soc Lond B Biol Sci 360:781-795.

Petrides M, Pandya DN (2006) Efferent association pathways originating in the caudal prefrontal cortex in the macaque monkey. J Comp Neurol 498:227-251.

Rockland KS (1997) Elements of cortical architecture: hierarchy revisited. In: Cerebral cortex (Rockland KS, Kadmon G, Peters A, eds), pp 243-294. New York: Plenum.

Romo R, Salinas E (2003) Flutter discrimination: neural codes, perception, memory and decision making. Nat Rev Neurosci 4:203-218.

Shafi M, Zhou Y, Quintana J, Chow C, Fuster J, Bodner M (2007) Variability in neuronal activity in primate cortex during working memory tasks. Neuroscience 146:1082-1108.

Vigneswaran G, Kraskov A, Lemon RN (2011) Large identified pyramidal cells in macaque motor and premotor cortex exhibit "Thin Spikes": implications for cell type classification. J Neurosci 31:14235-14242.

Wallis JD, Miller EK (2003) From rule to response: neuronal processes in the premotor and prefrontal cortex. J Neurophysiol 90:1790-1806.

Wang XJ (2001) Synaptic reverberation underlying mnemonic persistent activity. Trends Neurosci 24:455-463.

Zaksas D, Pasternak T (2006) Directional signals in the prefrontal cortex and in area MT during a working memory for visual motion task. J Neurosci 26:11726-11742. 